\title{
A Comparative Linear and Nonlinear Stability Analysis of Hybrid Journal Bearing Operating with Micropolar Lubricant
}

\author{
Kamal Budheeja* and Suresh Verma \\ 1)Department of Mechanical Engineering, Deenbandhu Chhotu Ram University of Science \& Technology, \\ Murthal, Sonepat, Haryana 131039, India \\ "Corresponding author: Kamal Budheeja (kamal.budheeja@gmail.com)
}

Manuscript received 24 January 2017; accepted 02 July 2017; published 15 October 2017

\begin{abstract}
The present study discusses the effect of micropolar parameters on the stability of symmetric hole-entry hybrid journal bearing system compensated with capillary restrictor. Finite element method has been used to solve the modified Reynolds equation for the flow of micropolar lubricant through the bearing. The linearized and nonlinear equations of motion are solved numerically using fourth order Runge-Kutta Method at each integration time step. Solution of these equations provides the locus of moving journal center and the journal trajectories are plotted with the help of a computer program. A Routh-Hurwitz stability criterion has been applied to calculate the critical mass of the journal to analyze the stability of journal. A comparative analysis for linear and nonlinear models considering Newtonian and micropolar lubricants has been done. The results obtained indicate that micropolar parameters affect the performance as well as the stability margin of the hybrid journal bearing system considerably and nonlinear analysis provides quick response as compared to linear one.
\end{abstract}

\section{Keywords}

capillary, micropolar lubricant, hybrid journal bearing, symmetric, hole-entry, finite element method, stability, linear, nonlinear

\section{Introduction}

Hole-entry hybrid journal bearings were developed to give good performance than the recessed or pocketed journal bearings due to less hydrodynamic action in the latter. A hole-entry hybrid journal bearing is used to operate at very heavy load and higher speed. In past years, many studies related to performance characteristics of non-recessed journal bearings have been carried out and reported in literature. In a study concerning different geometries of hybrid journal bearings, it was concluded by Row et al. [1] that the performance of non-recessed journal bearings is better than that of recessed bearings. A study related to selection of bearing configurations, geometry, flow control devices and materials was reported by Stout and Rowe [2], in which they found non-recessed bearings better; to suit high speed applications. Non-recessed hybrid journal bearing systems have received tremendous attention due to characteristics like large fluid-film stiffness \& damping, improved tolerance to manufacturing errors, high load-carrying capacity etc. These characteristics made them applicable to various engineering applications like precision grinding spindles, liquid rocket pumps, high speed turbo-machinery etc. [3].

Effective lubrication in journal bearings has become an important aspect for good performance of the bearings in the recent years. Various additives are generally added to Newtonian lubricants to improve the performance. Inadequacy of Newtonian lubricants to estimate the accurate flow behavior resulted in application of micropolar lubricants to solve lubrication problems. The concept of micropolar fluid theory was first introduced by Eringen [4], who discussed that each particle of lubricant is rigid \& non-deformable and possesses three rotational and three translational degrees of freedom. Since then many studies pertaining to micropolar lubricants are reported in literature. For example, the effect of micropolar fluids on the lubrication performance of misaligned hydrodynamic journal bearings was investigated by Das et al. [5] and it was observed that load carrying capacity increases in case of micropolar lubricant. Wang et al. [6] studied the lubricating effectiveness of micropolar fluids in a dynamically loaded journal bearing and found that micropolar fluids yield a higher oil film pressure and oil thickness compared to Newtonian fluids when subjected to same dynamic loading conditions. Wang et al. [7] discussed that micropolar fluids exhibit increase in load capacity and temperature and decrease in coefficient of friction and side leakage flow as compared to Newtonian fluids. An analysis of capillary compensated hydrostatic journal bearing 
operating with micropolar lubricant was carried out by Verma et al. [8]. Sharma et al. [9] performed a study on slot-entry hybrid journal bearing operating with Micropolar lubricant. An analysis of orifice compensated non-recessed hole-entry hybrid journal bearing operating with micropolar lubricants was done by Ram et al. [10] to obtain optimum values of fluid film stiffness coefficients. Verma et al. [11,12] analyzed and compared performance characteristics of flexible multirecess capillary and CFV compensated hydrostatic journal bearing operating with micropolar lubricant and observed a significant influence of bearing shell flexibility and micropolar effect on bearing performance. Recent studies on journal bearings operating with micropolar lubricant by Ram et al. [13] and Rana et al. [14] demonstrated the influence of micropolar parameters on performance characteristics of journal bearings.

Since the stability of journal bearing system is of prime importance in bearings, many studies concerning the stability analysis of rotating journal have been carried out. A computer aided study of the transient response of a two-lobe journal bearing with non-Newtonian lubricant by Sinhasan et al. [15] concluded that the nonlinear trajectories obtained indicated instability even at journal mass less than critical mass. Andreas [16] presented a computational analysis for the prediction of the transient dynamics of a rigid rotor supported in externally pressurized turbulent flow fluid film bearings. Turaga et al. [17] performed non-linear transient stability analysis to study the sub-synchronous whirl stability of a rigid rotor supported on two symmetric hydrodynamic bearings with rough surfaces subjected to a unidirectional constant load. Das et al. [18] performed a linear stability analysis of hydrodynamic journal bearings under micropolar lubrication, in which dynamic characteristics like stiffness and damping coefficients, critical mass parameter, whirl ratio etc. were obtained with respect to micropolar parameters. Nagaraju et al. [19] in their study found the stability margin of a roughened hole-entry orifice compensated hybrid journal bearing system. A nonlinear dynamic analysis of a flexible rotor supported by micropolar fluid film journal bearings was done by Chan-Jian et al. [20]. Kushare et al. [21] demonstrated in their theoretical study, how the non-Newtonian behavior of the lubricant has profound influence on journal trajectories and stability of a worn hybrid journal bearing. Recently, Mehrjardi et al. [24] did a comparative study of the preload effects on the stability performance of noncircular journal bearings using linear and nonlinear dynamic approaches.

A thorough scan of the literature indicates that micropolar lubricants enhances the performance characteristics of hybrid journal bearing system and nonlinear analysis set the correct stability margin as compared to linear analysis. Even nonrecessed bearings have advantages and preference over pocketed journal bearings, thus, linear and nonlinear models for plotting journal motion trajectories have been used in the present study to analyze the stability margin of hole-entry capillary compensated hybrid journal bearing system to make a comparative analysis among the two models. Trajectories are plotted for a hybrid journal bearing system operating with Newtonian as well as micropolar lubricant to make a comparison. Since state of the art technologies demand exceptional performances, therefore, the present work investigates the influence of micropolar parameters on the stability margin of a capillary compensated symmetric hole-entry hybrid journal bearing. To carry out the stability

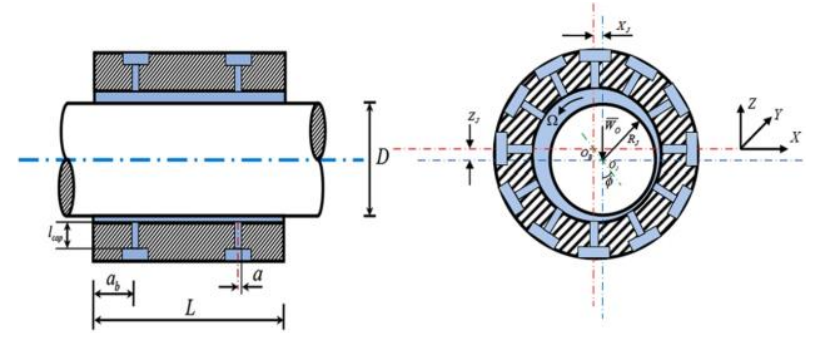

(a)

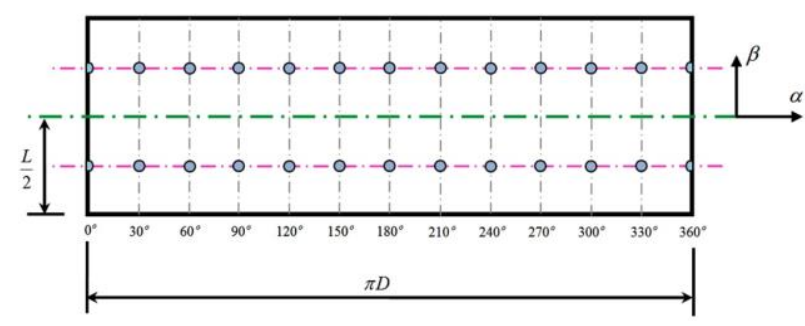

(b)

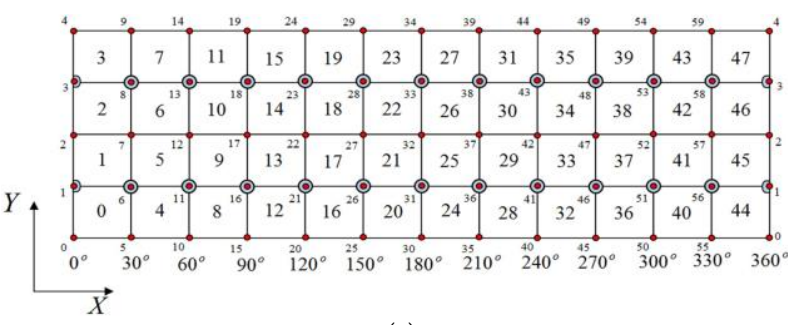

(c)

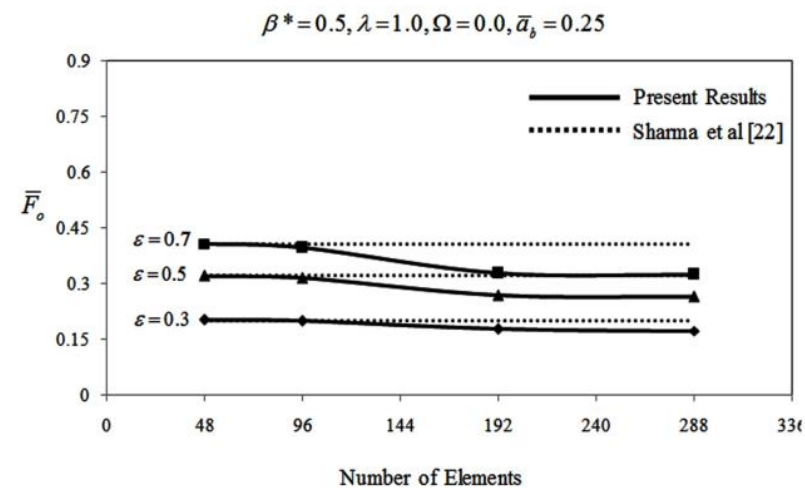

(d)

Fig. 1 (a) Symmetric Hole-Entry Hybrid Journal Bearing System (b) Geometry of Symmetric Hole-Entry Hybrid Journal Bearing System (c) Fluid film domain discretization using 4-noded quadrilateral isoparametric elements for finite element formulation of modified Reynold's equation (d) Fluid film reaction vs No. of elements at $\beta^{*}=0.5$, $\lambda=1.0, \quad \Omega=0.0, \quad \bar{a}_{\mathrm{k}}=0.25$

analysis, the static characteristics like fluid film pressure, minimum film thickness, load carrying capacity etc. and dynamic characteristics such as stiffness and damping coefficients are calculated first and then stability parameters vis o vis critical mass, threshold speed and frequency of whirl of the journal are determined. The novelty in the present work is to obtain and analyze the linear and nonlinear trajectories of the 
journal centre motion together from the transient stability point of view. In the present analysis, a symmetric rigid smooth hybrid journal bearing with 12 holes entry per row as shown in Figs. $1(a, b)$ is considered. The outcomes of the present work are expected to be quite useful for bearing designers and academic community.

\section{Governing equations}

The Modified Reynolds equation governing the flow of a micropolar lubricant in the convergent area of a hybrid journal bearing incorporating the micropolar parameters with usual assumptions of the lubrication film and constancy of the characteristics coefficients across the film of micropolar lubricant is given as [8]:

$$
\frac{\partial}{\partial x}\left(\frac{h^{3}}{12 \mu} \Phi \frac{\partial p}{\partial x}\right)+\frac{\partial}{\partial y}\left(\frac{h^{3}}{12 \mu} \Phi \frac{\partial p}{\partial y}\right)=\frac{\omega_{I} R_{I}}{2} \frac{\partial h}{\partial x}+\frac{\partial h}{\partial t}
$$

where

$$
\Phi=f(N, l, h)=h^{3}+12 h l^{2}-6 N l h^{2} \operatorname{coth}\left(\frac{N h}{2 l}\right)
$$

and all notations have their usual meanings.

The above equation can be written in dimensionless form as [8]:

$$
\frac{\partial}{\partial \alpha}\left(\frac{\bar{h}^{3}}{12} \frac{\bar{\Phi}}{\bar{\mu}} \frac{\partial \bar{p}}{\partial \alpha}\right)+\frac{\partial}{\partial \beta}\left(\frac{\bar{h}^{3}}{12} \frac{\bar{\Phi}}{\bar{\mu}} \frac{\partial \bar{p}}{\partial \beta}\right)=\frac{\Omega}{2} \frac{\partial \bar{h}}{\partial \alpha}+\frac{\partial \bar{h}}{\partial \bar{t}}
$$

where

$$
\bar{\Phi}=\left[1+\frac{12}{\bar{h}^{2} l_{m}^{2}}-\frac{6 N}{\bar{h} l_{m}} \operatorname{coth}\left(\frac{N \bar{h} l_{m}}{2}\right)\right]=f\left(N, l_{m}, h\right)
$$

$$
\bar{h}=\frac{h}{c} \quad \alpha, \beta=\frac{(x, y)}{R_{I}} \quad \bar{\mu}=\frac{\mu}{\mu_{r}} \quad \bar{t}=t\left[\frac{c^{2} p_{s}}{\mu_{r} R_{I}{ }^{2}}\right] \quad \bar{p}=\frac{p}{p_{s}}
$$

2.1 Fluid film thickness

The fluid-film thickness expression for a rigid symmetric hole-entry hybrid journal bearing system, in non-dimensional form is given as [8]:

$$
\bar{h}=\bar{h}_{o}+\Delta \bar{h}
$$

where $\Delta \bar{h}$ is the perturbation on the fluid-film thickness due to dynamic conditions.

$\bar{h}_{o}$ is the fluid-film thickness when journal center is in static equilibrium position and may be given as:

$$
\bar{h}_{o}=1-\bar{X}_{J} \cos \alpha-\bar{Z}_{J} \sin \alpha
$$

2.2 Restrictor flow equation

Hybrid journal bearings are used in conjunction with some restrictors or flow control devices like constant flow valve, capillary, orifice, slot etc. In the present work, capillary has been used as restrictor. The flow of micropolar lubricant through capillary restrictor in dimensionless form is given by [8]

$$
\bar{Q}_{R}=\bar{C}_{s 2}\left(1-\bar{p}_{c}\right)
$$

where

$$
\overline{\mathrm{C}}_{\mathrm{s} 2}=\frac{\pi a^{4}}{8 c^{3} l_{\text {cap }}},
$$

$\bar{p}_{c}=\frac{p_{c}}{p_{s}}$ is hole pressure.

\subsection{Boundary conditions}

The boundary conditions used for the solution of lubricant flow field are taken as [23]:

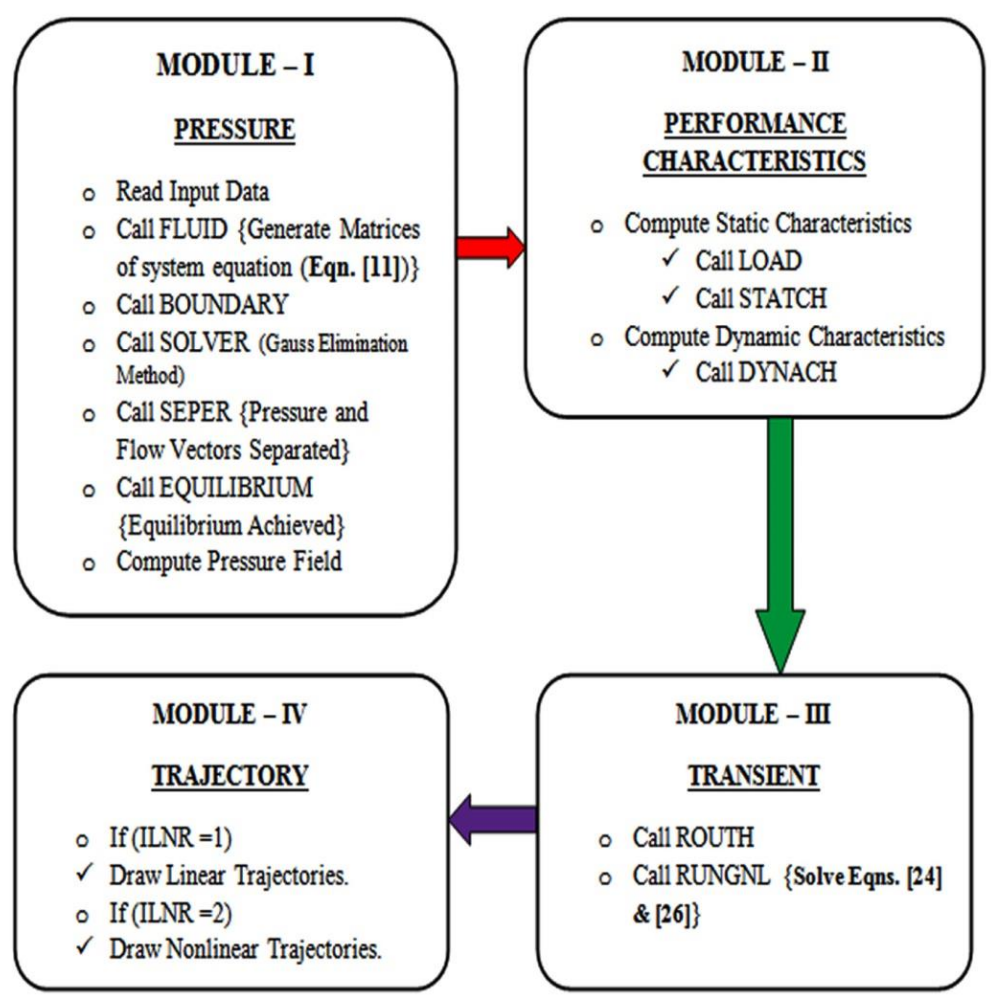

Fig. 2 Overall Iterative Solution Procedure 
- Nodes situated on the external boundary of the bearing have zero relative pressure with respect to atmospheric pressure.

$$
\left.\bar{p}\right|_{\beta=\mp \lambda}=0.0
$$

- Pressures are equal for nodes situated on centre of entry-hole position.

- The nodal flows are zero at internal nodes except those situated on holes and external boundaries.

- Flow of lubricant through the restrictor is equal to the bearing input flow.

\subsection{Finite element formulation}

The lubricant flow field in the clearance space of symmetric hole-entry hybrid journal bearing has been discretized using four-noded quadrilateral isoparametric elements. Flow field is discretized using 48 elements connected by 60 nodes. Numbering of nodes is done such that the bandwidth is minimum. Discretization scheme is shown in Fig. 1(c) in which elements are represented by element numbers written at middle of each element. The nodes are located at the corners of the elements and are represented by node numbers marked with red dots. The holes are represented by small circles indicated at nodes $1,3,6,8,11,13 \ldots . .$. so on.

For deciding the number of elements and nodes, combinations of 48 elements 60 nodes, 96 elements 108 nodes, 192 elements 216 nodes and 288 elements 312 nodes were used to find fluid film reaction $\bar{F}_{0}$ at different eccentricity ratios and $\beta^{*}=0.5, \lambda=1.0, \quad \Omega=0.0, \quad \bar{a}_{b}=0.25$. The present results were compared with the results presented in the work of Sharma et al [22]. The results were found very close to the published results when 48 elements and 60 nodes were selected as shown in Fig. 1(d).

Using Galerkin's Orthogonality conditions and following the usual assembly procedure, the global system of equation is obtained as [8]:

$$
[\bar{F}]\{\bar{p}\}=\{\bar{Q}\}+\Omega\left\{\bar{R}_{H}\right\}+\bar{X}_{I}\left\{\bar{R}_{\text {XI }}\right\}+\overline{\dot{Z}}_{J}\left\{\bar{R}_{\text {zl }}\right\}
$$

Each term of respective matrix / vector is computed using following expressions:

$$
\begin{aligned}
& {\left[\bar{F}_{i j}\right]^{e}=\iint_{\bar{A}_{e}}\left\{\frac{\bar{h}^{3}}{12 \bar{\mu}}\left(\bar{\Phi} \frac{\partial N_{i}}{\partial \alpha} \frac{\partial N_{j}}{\partial \alpha}+\bar{\Phi} \frac{\partial N_{i}}{\partial \beta} \frac{\partial N_{j}}{\partial \beta}\right)\right\} \partial \alpha \partial \beta} \\
& {\left[\bar{Q}_{i}\right]^{e}=\int_{\Gamma^{e}}\left\{\left(\frac{\bar{h}^{3}}{12 \bar{\mu}}\left(\bar{\Phi} \frac{\partial \bar{p}}{\partial \alpha}\right)-\frac{\bar{\Omega}}{2} \bar{h}\right) l_{1}+\frac{\bar{h}^{3}}{12 \bar{\mu}}\left(\bar{\Phi} \frac{\partial \bar{p}}{\partial \beta}\right) l_{2}\right\} N_{i} \mathrm{~d} \bar{\Gamma}_{e}} \\
& \bar{R}_{H i}^{e}=\iint_{\bar{A}_{e}}\left\{\frac{h}{2} \frac{\partial N_{i}}{\partial \alpha}\right\} \partial \alpha \partial \beta \\
& \bar{R}_{X j i}^{e}=\iint_{\bar{A}_{e}} \cos \alpha N_{i} \partial \alpha \partial \beta \\
& \bar{R}_{Z j i}^{e}=\iint_{\bar{A}_{e}} \sin \alpha N_{i} \partial \alpha \partial \beta
\end{aligned}
$$

where $l_{1}$ and $l_{2}$ are direction cosines and $i, j=1,2, \ldots \ldots \ldots . ., n_{l}^{e}$ (number of nodes per element).

\subsection{Fluid film reaction}

The fluid film reaction components are given as [17]:

$$
\begin{aligned}
& \bar{F}_{x}=-\int_{-\lambda}^{\lambda} \int_{0}^{2 \pi} \bar{p} \cos \alpha \mathrm{d} \alpha \mathrm{d} \beta \\
& \bar{F}_{z}=-\int_{-\lambda}^{\lambda} \int_{0}^{2 \pi} \bar{p} \sin \alpha \mathrm{d} \alpha \mathrm{d} \beta
\end{aligned}
$$

The resulting fluid film reaction is expressed as:

$$
\bar{F}_{o}=\left[\bar{F}_{x}^{2}+\bar{F}_{z}^{2}\right]^{\frac{1}{2}}
$$

\subsection{Dynamic fluid film coefficients}

The bearing dynamic coefficients i.e. stiffness and damping coefficient of fluid-film are considered to be the dynamic fluid film coefficients. For two degree of freedom system, there exist four stiffness and four damping coefficients, which can be used to study the stability of the system.

2.6.1 Fluid-film stiffness coefficients

The fluid-film stiffness coefficients are given as [12]:

$$
\bar{S}_{i j}=-\frac{\partial \bar{F}_{i}}{\partial q_{j}}(i=x, z)
$$

where ' $i$ ' represents the direction of force and $q_{j}=\bar{X}_{J}, \bar{Z}_{J}$

In Matrix Form:

$$
\left[\begin{array}{ll}
\bar{S}_{x x} & \bar{S}_{x z} \\
\bar{S}_{z x} & \bar{S}_{z z}
\end{array}\right]=-\left[\begin{array}{ll}
\frac{\partial \bar{F}_{x}}{\partial \bar{X}_{I}} & \frac{\partial \bar{F}_{x}}{\partial \bar{Z}_{I}} \\
\frac{\partial \bar{F}_{z}}{\partial \bar{X}_{J}} & \frac{\partial \bar{F}_{z}}{\partial \bar{Z}_{I}}
\end{array}\right]
$$

2.6.2 Fluid-film damping coefficients:

The fluid-film damping coefficients are given as [12]

$$
\bar{C}_{i j}=-\frac{\partial \bar{F}_{i}}{\partial \dot{q}_{j}}(i=x, z)
$$

where ' $i$ ' represents the direction of force and $\dot{q}_{j}=\overline{\dot{X}}_{J}, \bar{Z}_{J}$ represents the velocity component of journal center.

In Matrix Form:

$$
\left[\begin{array}{ll}
\bar{C}_{x x} & \bar{C}_{x z} \\
\bar{C}_{z x} & \bar{C}_{z z}
\end{array}\right]=-\left[\begin{array}{ll}
\frac{\partial \bar{F}_{x}}{\partial \dot{X}_{I}} & \frac{\partial \bar{F}_{x}}{\partial \dot{Z}_{I}} \\
\frac{\partial \bar{F}_{z}}{\partial \dot{\bar{X}}_{I}} & \frac{\partial \bar{F}_{z}}{\partial \overline{\dot{Z}}_{I}}
\end{array}\right]
$$

\subsection{Journal centre motion trajectory}

Journal centre starts whirling about its static equilibrium position as soon as journal is perturbed from its equilibrium position due to change in hydrodynamic forces. The transient response of a journal bearing system under these conditions can be obtained either by linear or nonlinear equation of motion. Thus, the locus of instantaneous journal center position i.e. journal motion trajectory may be obtained by numerically integrating linearized and nonlinear equations of motion of journal. In simple terms, the term "linear trajectory" and "nonlinear trajectory" refers to the trajectory when it is obtained from linearized or nonlinear equation of motions respectively.

2.7.1 Linearized equations of motion

The linearized equation of motion for the journal may be given as [21]:

$$
\left[\begin{array}{cc}
\bar{M}_{I} & 0 \\
0 & \bar{M}_{I}
\end{array}\right]\left\{\begin{array}{l}
\overline{\ddot{X}}_{I} \\
\bar{Z}_{J}
\end{array}\right\}+\left[\begin{array}{ll}
\bar{C}_{x x} & \bar{C}_{x z} \\
\bar{C}_{z x} & \bar{C}_{z z}
\end{array}\right]\left\{\begin{array}{l}
\overline{\dot{X}}_{I} \\
\overline{\bar{Z}}_{J}
\end{array}\right\}+\left[\begin{array}{ll}
\bar{S}_{x x} & \bar{S}_{x z} \\
\bar{S}_{z x} & \bar{S}_{z z}
\end{array}\right]\left\{\begin{array}{l}
\bar{X}_{I} \\
\bar{Z}_{I}
\end{array}\right\}=0
$$

2.7.2 Nonlinear equations of motion

The nonlinear equations of disturbed journal motion in terms of instantaneous fluid-film force components $\bar{F}_{x}$ and $\bar{F}_{z}$ at any time $\bar{t}$ may be written as [21]: 


$$
\left[\begin{array}{cc}
\bar{M}_{J} & 0 \\
0 & \bar{M}_{J}
\end{array}\right]\left\{\begin{array}{l}
\bar{X}_{J} \\
\bar{Z}_{J}
\end{array}\right\}=\left\{\begin{array}{l}
\bar{F}_{x}-\bar{F}_{o x} \\
\bar{F}_{z}-\bar{F}_{o z}
\end{array}\right\}
$$

where $\bar{F}_{x}$ and $\bar{F}_{z}$ are the hydrodynamic components of fluid film forces in $x$ and $z$ directions respectively and are nonlinear functions of the displacement component $\left(\bar{X}_{J}, \bar{Z}_{J}\right)$ and the velocities $\left(\overline{\dot{X}}_{J}, \overline{\dot{Z}}_{J}\right)$ of the journal center. They are calculated by integrating the pressure over the bearing surface. $\bar{F}_{o x}$ and $\bar{F}_{o z}$ are the fluid-film force components at the static equilibrium position.

$$
\text { Thus, }\left[\begin{array}{cc}
\bar{M}_{I} & 0 \\
0 & \bar{M}_{I}
\end{array}\right]\left\{\begin{array}{l}
\bar{X}_{I} \\
\bar{Z}_{I}
\end{array}\right\}=\left\{\begin{array}{l}
\Delta \bar{F}_{x} \\
\Delta \bar{F}_{z}
\end{array}\right\}
$$

\subsection{Stability parameters}

Equations (24) and (26) are numerically integrated using $4^{\text {th }}$ Order Runge Kutta Method for certain initial disturbance
$\left(\bar{X}_{J}, \bar{Z}_{J}\right)$ \& $\left(\overline{\dot{X}}_{J}, \overline{\dot{Z}}_{J}\right)$ to give new $\left(\bar{X}_{J}, \bar{Z}_{J}\right)$ \& $\left(\overline{\dot{X}}_{J}, \overline{\dot{Z}}_{J}\right)$ which would ultimately be used to draw the linear and nonlinear journal trajectories. Routh's Stability criterion is used to find the stability parameters (in terms of critical mass of journal, Threshold speed and Frequency of whirl) of the journal. Dimensionless critical mass of journal Bearing is given as [21]:

$$
\bar{M}_{c}=\frac{\bar{\Delta}_{1}}{\bar{\Delta}_{2}-\bar{\Delta}_{3}}
$$

where

$$
\begin{aligned}
& \bar{\Delta}_{1}=\left[\bar{C}_{x x} \bar{C}_{z z}-\bar{C}_{z x} \bar{C}_{x z}\right] \\
& \bar{\Delta}_{2}=\frac{\left[\bar{S}_{x x} \bar{S}_{z z}-\bar{S}_{x z} \bar{S}_{z x}\right]\left[\bar{C}_{x x}+\bar{C}_{z z}\right]}{\left[\bar{S}_{x x} \bar{C}_{z z}+\bar{S}_{z z} \bar{C}_{x x}-\bar{S}_{x z} \bar{C}_{z x}-\bar{S}_{z x} \bar{C}_{x z}\right]} \\
& \bar{\Delta}_{3}=\frac{\left[\bar{S}_{x x} \bar{C}_{x x}+\bar{S}_{x z} \bar{C}_{x z}+\bar{S}_{z z} \bar{C}_{z z}\right]}{\left[\bar{C}_{x x}+\bar{C}_{z z}\right]}
\end{aligned}
$$

Threshold speed and frequency of whirl can be obtained by following relations:

Threshold speed,

$$
\bar{\omega}_{t h}=\left(\frac{\bar{M}_{c}}{\bar{F}_{o}}\right)^{\frac{1}{2}}
$$

where $\bar{F}_{o}$ is the resultant fluid film reaction.

Frequency of whirl,

$$
f_{w}=\sqrt{\frac{1}{\bar{M}_{c}\left(\frac{\bar{S}_{x x} \bar{C}_{z z}+\bar{S}_{z z} \bar{C}_{x x}-\bar{S}_{x z} \bar{C}_{z x}-\bar{S}_{z x} \bar{C}_{x z}}{\bar{C}_{x x}+\bar{C}_{z z}}\right)}}
$$

\section{Solution procedure}

The solution procedure involves four modules namely PRESSURE, PERFORMANCE CHARACTERISTICS, TRANSIENT and TRAJECTORY. 2D Input Data is read from input data file and initial fluid film thickness is calculated using Eqn. (6), consequently Module-I (PRESSURE) becomes operative.
Nodal pressures are then calculated, as sub-modules FLUID, BOUNDARY, SOLVER, SEPER and EQUILIBRIUM in Module I (PRESSURE) operates simultaneously and iteratively to generate matrices of system equation (Eqn. (11)) after modifying system equation according to boundary conditions, applying Gauss Elimination Method to solve system equation, separating pressure and flow vectors and achieving journal centre equilibrium respectively. Fluid film reaction components are then computed using Eqns. ((17) and (18)) and then static and dynamic characteristics are calculated by sub-modules LOAD, STATCH and DYNACH respectively under Module-II (PERFORMANCE CHARACTERISTICS). Stability parameters are obtained by solving Eqns. (27-31) in sub-module ROUTH, as well as linear and nonlinear equations of journal motion are solved in sub-module RUNGNL in Module-III (TRANSIENT). These equations of motion are numerically integrated iteratively with initial values of disturbances $\left(\bar{X}_{J}, \bar{Z}_{J} ; \overline{\dot{X}}, \overline{\dot{Z}}\right)$ by fourth order Runge Kutta Method to give new journal displacements and velocities for next time step. The initial values of displacements

Table 1 Non-dimensional Operating and Geometric Parameters for Symmetric Hybrid Journal Bearing System

\begin{tabular}{|c|c|c|c|}
\hline \multicolumn{2}{|c|}{ Operating Parameters (Symmetric) } & \multicolumn{2}{c|}{ Geometric Parameters (Symmetric) } \\
\hline Parameters & Values/Range & Parameters & Value \\
\hline External Load $\left(\bar{W}_{o}\right)$ & $1.0-4.0$ & No. of Rows & 02 \\
\hline Speed Parameter $(\Omega)$ & 1.0 & No. of Holes per Row & 12 \\
\hline Capillary - Restrictor Design Parameter $\left(\bar{C}_{s 2}\right)$ & $0.05-0.25$ & Aspect Ratio $(\lambda)$ & 1.0 \\
\hline Characteristic Length $\left(l_{m}\right)$ & $10-50$ & Land Width Ratio $\left(\bar{a}_{b}\right)$ & 0.25 \\
\hline Coupling Number $\left(N^{2}\right)$ & $0.1-0.9$ & Clearance Ratio $(\bar{c})$ & 0.001 \\
\hline \multicolumn{2}{|c|}{ For Newtonian Lubricant: $N^{2} \rightarrow 0.0, l_{m} \rightarrow \infty$} \\
\hline
\end{tabular}


Table 2 Dimensional Values of Operating and Geometric Parameters for Symmetric Hybrid Journal Bearing System

\begin{tabular}{|c|c|c|}
\hline Parameter & Notation & Value \\
\hline Radial Clearance & $c$ & $0.0502 \times 10^{-3} \mathrm{~m}$ \\
\hline Journal Radius & $R_{I}$ & $0.05 \mathrm{~m}$ \\
\hline Bearing Length & $L$ & $0.1 \mathrm{~m}$ \\
\hline Load & $W_{o}$ & $22.4 \mathrm{kN}$ \\
\hline Lubricant Viscosity & $\mu$ & $0.0345 \mathrm{Nsm}^{-2}$ at $38^{\circ} \mathrm{C}$ \\
\hline Supply pressure & $p_{s}$ & $8.96 \mathrm{MNm}^{-2}$ \\
\hline Restrictor Flow & $Q_{c}$ & $32.8549 \times 10^{-6} \mathrm{~m}^{3} \mathrm{~s}^{-1}$ \\
\hline Journal Speed & $N_{o}$ & $2500 \mathrm{rpm}$ \\
\hline
\end{tabular}

and velocities of the journal centre are taken as $\left(\bar{X}_{J}=\bar{Z}_{I}=0.05\right)$ and $(\overline{\dot{X}}=\overline{\dot{Z}}=0.0)$ and a time step of $(\Delta \bar{t}=0.005)$ is taken for all the cases studied and analyzed. Finally, in Module-IV (TRAJECTORY), a series of journal displacement data is plotted to give locus of journal motion trajectory as per the case of linearized or nonlinear equations of motion.

The non-dimensional and dimensional bearing operating and geometric parameters are the most judiciously selected values based on the published literature $[8,12,21$ and 22] as shown in Table 1 and Table 2.

\section{Results and discussions}

The linear and nonlinear journal center motion trajectories of symmetric hole-entry hybrid journal bearing system, compensated with capillary restrictor and operating under micropolar lubrication have been drawn and analyzed. In order to check the validity of the developed simulation model of present study, load is computed at different eccentricity ratios for rigid hydrodynamic bearing operating with Newtonian and micropolar lubricants. The results obtained from present work have been compared with the available theoretical results of Wang et al. [7] and found to be very close as shown in Fig. 3. The difference in the result values may be attributed to the different computational scheme used. Further, the results for a smooth rigid capillary compensated symmetric hole-entry hybrid journal bearing are compared and contrasted against other proven results by Sharma et al. [22]. The comparison is listed in Table 3 and bears a remarkable resemblance.

The fluid film pressure profile over the circumference of the bearing operating at $\Omega=1.0, \quad \lambda=1.0, \quad \bar{a}_{b}=0.25, \quad \bar{C}_{s 2}=0.10$, $\bar{M}_{J}=\bar{M}_{C}$ and external load $\bar{W}_{o}=1.0$ and $\bar{W}_{o}=2.0$ is shown in Figs. $4(\mathrm{a}, \mathrm{b})$ respectively. It is observed that pressure increases with increase in micropolar effect in both linear and nonlinear cases. However, pressure profile in nonlinear case is always below the linear case for both Newtonian and micropolar fluids. From Fig. 4(a) it is observed that at lower load $\left(\bar{W}_{o}=1.0\right)$ cavitation is observed between $\alpha=120$ to $270^{\circ}$ in nonlinear case only. In Fig. 5, variation of attitude angle $(\phi)$ has been shown against external load $\left(\bar{W}_{o}\right)$, restrictor design parameter $\left(\bar{C}_{S 2}\right)$ and micropolar parameters $\left(N^{2}, l_{m}\right)$. Attitude angle initially increases with increase in load and then gradually decreases and it is maximum at $\bar{W}_{o}=2.0$ for Newtonian as well as micropolar fluids as observed from Fig. 5(a). On the other hand, while varying $\bar{C}_{s 2}$ from 0.05 to 0.25 in Fig. 5(b), attitude angle $(\phi)$ initially decreases and then a marginal increase in its value is observed. Moreover, it is also observed that attitude angle $(\phi)$ increases with increase in micropolar effect as evident from Fig. $5(\mathrm{c})$.

In Figs. 6-8, dashed trajectories indicate linear trajectories while dark trajectories indicate nonlinear trajectories. Figs. 6(a-c), $7(\mathrm{a}-\mathrm{c}), 8(\mathrm{a}-\mathrm{c})$ represent the trajectories plotted for Newtonian lubricant whereas Figs. 6(d-f), 7(d-f), 8(d-f) represent the trajectories plotted for micropolar lubricant. Linear and nonlinear journal motion trajectories at different masses of journal $\quad \bar{M}_{J}=0.80 \bar{M}_{C^{\prime}} \quad \bar{M}_{J}=1.0 \bar{M}_{C^{\prime}} \quad \bar{M}_{J}=1.1 \bar{M}_{C} \quad$ at load $\bar{W}_{o}=1.0$, restrictor design parameter $\bar{C}_{s 2}=0.10$ for Newtonian and micropolar lubricants have been drawn for stability analysis of circular symmetric hole-entry journal bearing compensated with capillary restrictor in Figs. 6(a-f). It is observed from Figs. $6(a, d)$ that linearized equations of motion predicts the stable cycle for both Newtonian and micropolar lubricants, when $\bar{M}_{I}=0.80 \bar{M}_{C}$, i. e., when the mass of the journal is less than critical mass, but nonlinear equations of motion predicts unstable cycle for same operating parameters. The reason for this instability may be attributed to the fact that journal operates at

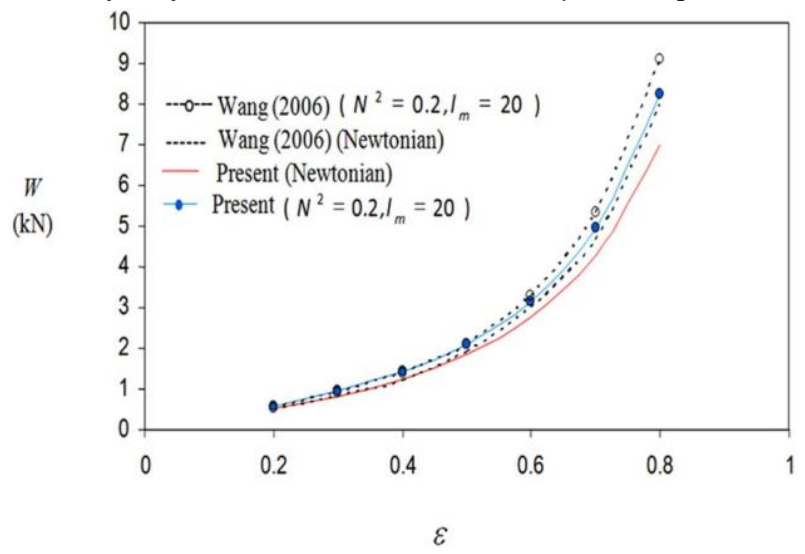

Fig. 3 Variation of load carrying capacity $W(\mathrm{kN})$ with eccentricity ratio $(\varepsilon)$ for rigid hydrodynamic journal bearing

Tribology Online, Vol. 12, No. 5 (2017) / 208 
Table 3 Validation Table

$\bar{C}_{s 2}=0.087267, \Omega=1.0, \bar{a}_{b}=0.25, \lambda=1.0, \beta^{*}=0.5$

\begin{tabular}{|c|c|c|}
\hline \multirow{2}{*}{$\begin{array}{l}\text { STATIC \& DYNAMIC } \\
\text { CHARACTERISTICS }\end{array}$} & \multicolumn{2}{|c|}{$\bar{W}_{o}$} \\
\hline & 1.0 & 1.4 \\
\hline$h_{\min }[P]$ & 0.82872 & 0.76119 \\
\hline$h_{\min }[R]$ & 0.8288 & 0.7613 \\
\hline$\%$ age diff & -0.0095328 & -0.0145824 \\
\hline$S_{22}[P]$ & 2.86114 & 2.864 \\
\hline$S_{22}[R]$ & 2.863 & 2.862 \\
\hline$\%$ age diff & -0.065009 & 0.0698324 \\
\hline$C_{11}[P]$ & 10.7447 & 11.021 \\
\hline$C_{11}[R]$ & 10.725 & 11.0431 \\
\hline \%age diff & 0.1833462 & -0.2005263 \\
\hline$\omega_{t h}[P]$ & 3.37526 & 2.84194 \\
\hline$\sigma_{t h}[R]$ & 3.372 & 2.84 \\
\hline$\%$ age diff & 0.096585 & 0.068263 \\
\hline
\end{tabular}

$[P]-$ Present Results

$[R]$ - Reference Results (22)

$\%$ age diff $=\frac{\text { Present } \text { Results }- \text { Reference Results }}{\text { Present Results }} \times 100$

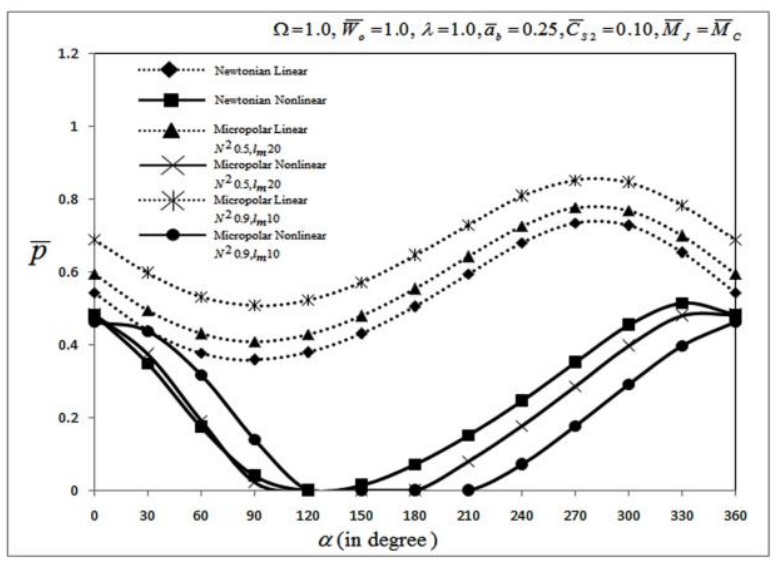

(a)

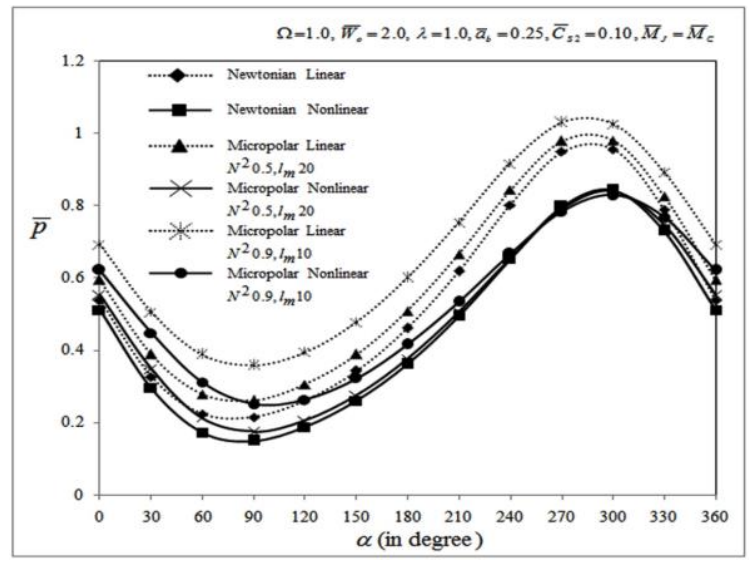

(b)

Fig. 4 Circumferential pressure profile of Symmetric Hole-Entry Hybrid Journal Bearing System operating with $\Omega=1.0, \quad \lambda=1.0$,

$$
\bar{a}_{b}=0.25, \quad \bar{C}_{s 2}=0.10, \quad \bar{M}_{I}=\bar{M}_{C} \text { at: (a) } \bar{W}_{o}=1.0, \text { (b) } \bar{W}_{o}=2.0
$$

high speed and lower load leading to higher radial clearance; consequently more lubricant is pumped in causing instability of the journal. When journal mass equals critical mass i.e. at $\bar{M}_{I}=1.0 \bar{M}_{C}$, journal motion trajectories obtained by linearized equations of motion form limit cycle for Newtonian and micropolar lubricants while nonlinear journal motion trajectories form unstable cycle as shown in Figs. 6(b,e). Instability in nonlinear case is seen due to instantaneous forces produced, which displace the journal from the boundary of limit cycle towards unstable region. When $\bar{M}_{J}=1.1 \bar{M}_{C}$, Figs. $6(\mathrm{c}, \mathrm{f})$ show that linear and nonlinear trajectories form unstable cycle for Newtonian as well as micropolar lubricant, but nonlinear trajectories diverge very fast. Further, it is noticed that the trajectories' start point shifts a little closer towards the origin in case of micropolar lubricant. Thus, it may be concluded from the results obtained that at lower loads, linear trajectory shows stability, limit cycle as well as instability at $\bar{M}_{J}=0.80 \bar{M}_{C}$, $\bar{M}_{J}=\bar{M}_{C^{\prime}} \quad \bar{M}_{J}=1.1 \bar{M}_{C}$ respectively while nonlinear trajectory shows instability in all cases for Newtonian as well as micropolar lubricants.

It is observed that linear and nonlinear trajectories form stable cycle at $\bar{W}_{o}=2.0, \quad \bar{C}_{s 2}=0.10$, in case of Newtonian and as well as micropolar lubricant at $\bar{M}_{J}=0.80 \bar{M}_{C}$, as shown in 


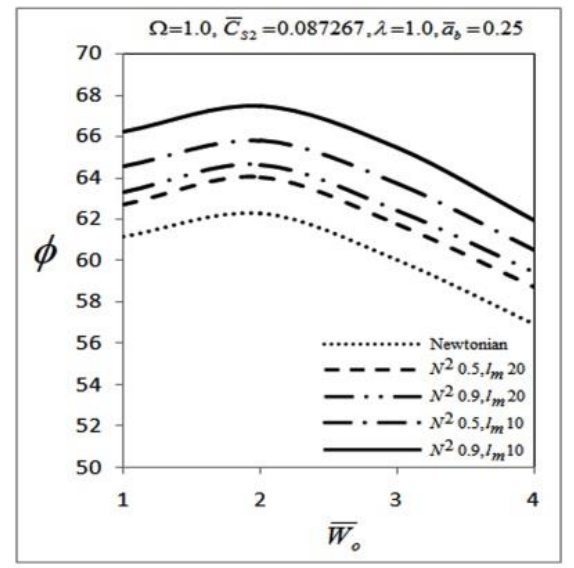

(a)

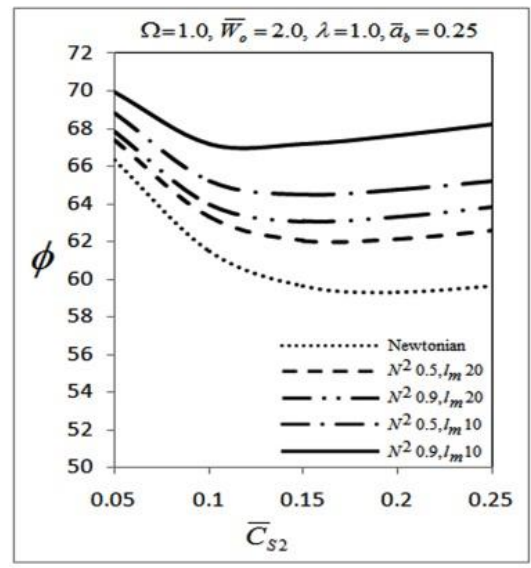

(b)

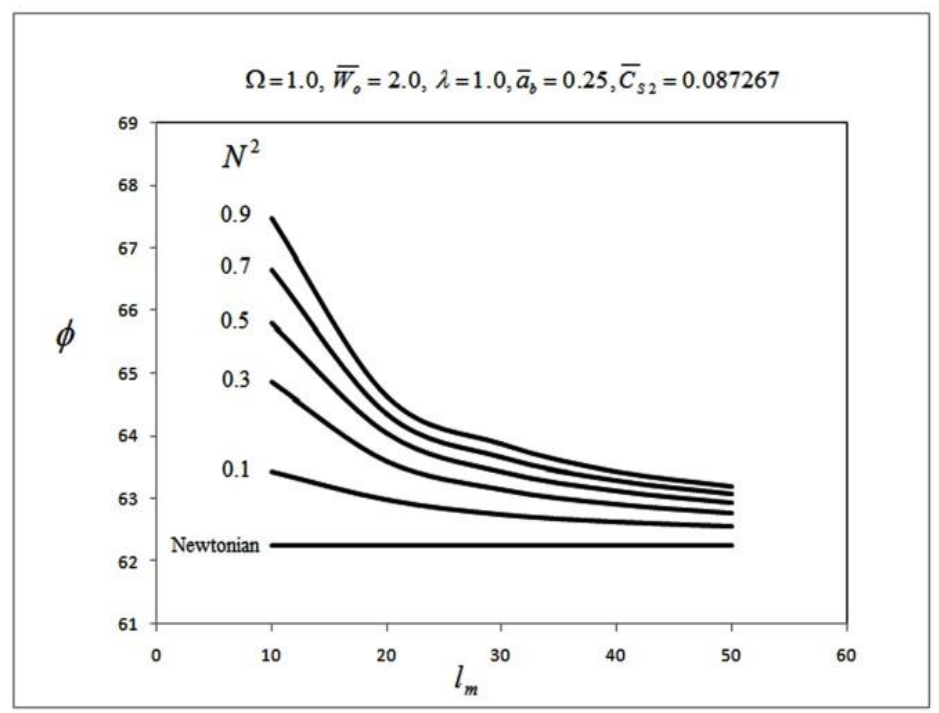

(c)

Fig. 5 Variation of attitude angle with (a) External load $\left(\bar{W}_{o}\right)$, (b) Restrictor design parameter $\left(\bar{C}_{s 2}\right)$, (c) Micropolar parameters $\left(N^{2}, l_{m}\right)$

Figs. 7(a,d). This is due to the fact that increased load balance the unbalanced hydrodynamic forces. When $\bar{M}_{I}=1.0 \bar{M}_{C^{\prime}}$ Figs. $7(\mathrm{~b}, \mathrm{e})$ depict that the linear trajectories exhibit limit cycle whereas the nonlinear trajectories show unstable motion. This is because when the nonlinear motion is considered, the instantaneous unbalanced hydrodynamic forces cause the journal to exit limit cycle and become unstable. As the mass of journal is taken above critical journal mass i.e. when $\bar{M}_{I}=1.1 \bar{M}_{C^{\prime}}$ the linear and nonlinear trajectories form unstable cycle for Newtonian and micropolar lubricants, shown in Figs. $7(\mathrm{c}, \mathrm{f})$.

For $\bar{W}_{o}=4.0, \quad \bar{C}_{s 2}=0.10$, in Figs. $8(\mathrm{a}, \mathrm{d})$ it is found that when $\bar{M}_{J}=0.80 \bar{M}_{C}$, linear and nonlinear trajectories form stable cycle in case of Newtonian and micropolar lubricants because of the components of load acts in a manner to balance the unbalanced forces whereas when $\bar{M}_{J}=1.0 \bar{M}_{C^{\prime}}$ the linear trajectories exhibit limit cycle and the nonlinear trajectories show stable motion as shown in Figs. $8(\mathrm{~b}, \mathrm{e})$. When $\bar{M}_{I}=1.1 \bar{M}_{C^{\prime}}$ the linear trajectories exhibit unstable motion and nonlinear trajectories form stable cycle for Newtonian and micropolar lubricants as shown in Figs. 8(c,f). The stability obtained in nonlinear cases is the result of very high load; which cause minimum radial clearance resulting in less lubricant flow in the clearance space, so unbalanced forces causing instability of the journal are minimized and balanced against heavy load. At heavy loads, the size of the orbit flattens and its size reduces in case of Newtonian but remains approximately circular in case of micropolar lubricant. Thus, it may be concluded that at heavy loads, linear trajectory shows stability, limit cycle and instability at $\quad \bar{M}_{J}=0.80 \bar{M}_{C^{\prime}} \quad \bar{M}_{J}=\bar{M}_{C^{\prime}} \quad \bar{M}_{J}=1.1 \bar{M}_{C}$ respectively while nonlinear trajectory shows stability in all cases for Newtonian as well as micropolar lubricants. The same effect of micropolar lubricant is observed at heavy loads also that trajectories shifts towards origin.

Figure 9 shows the influence of micropolar parameters on the journal motion trajectories at $\bar{W}_{0}=4.0, \Omega=1.0, \quad \bar{C}_{s 2}=0.25$, $\bar{M}_{I}=\bar{M}_{C}$. It is observed that linear trajectories show limit cycle 


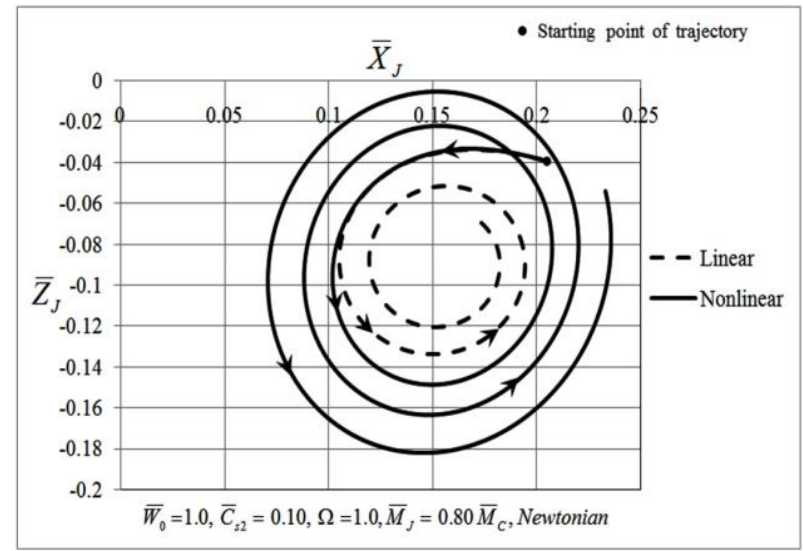

(a)

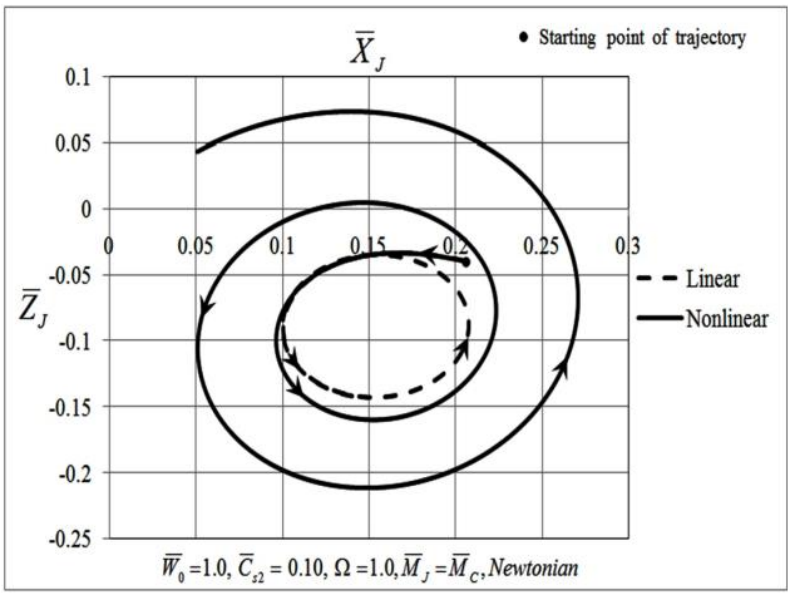

(b)

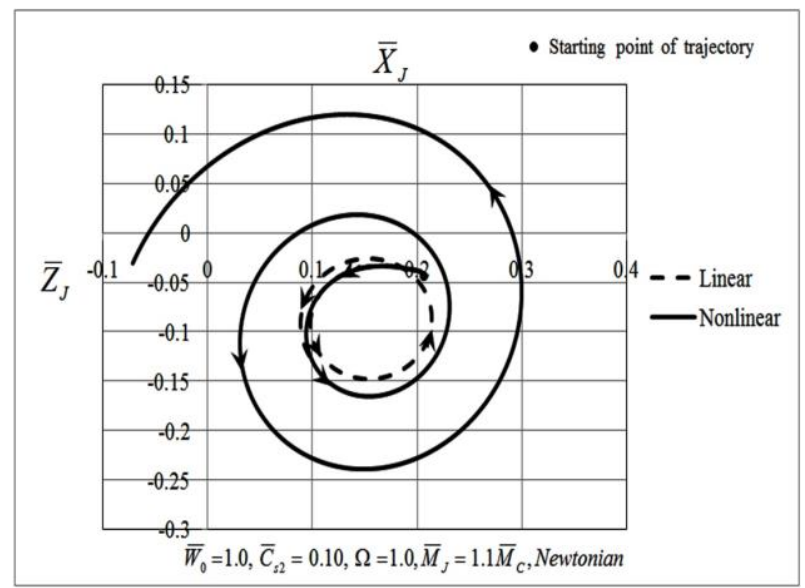

(c)

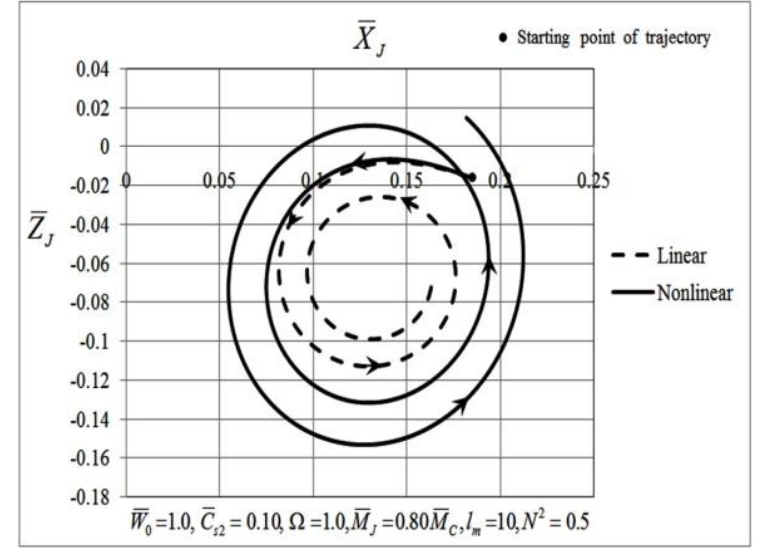

(d)

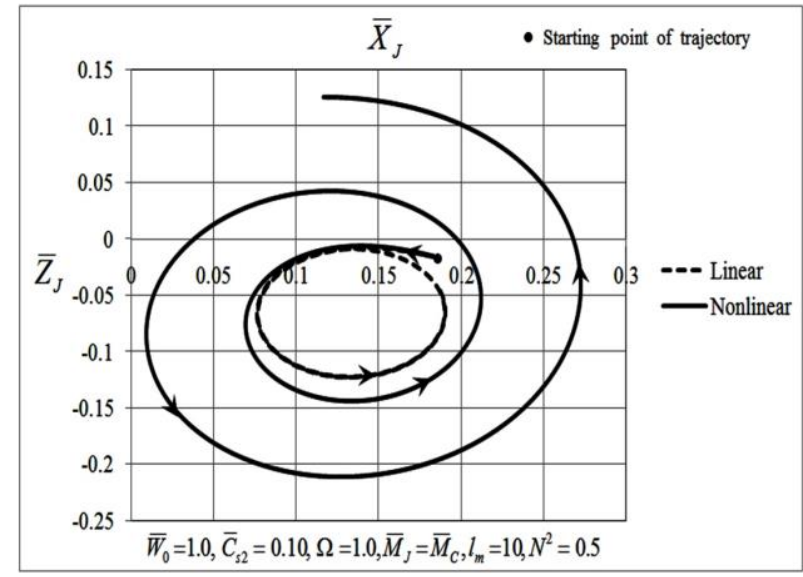

(e)

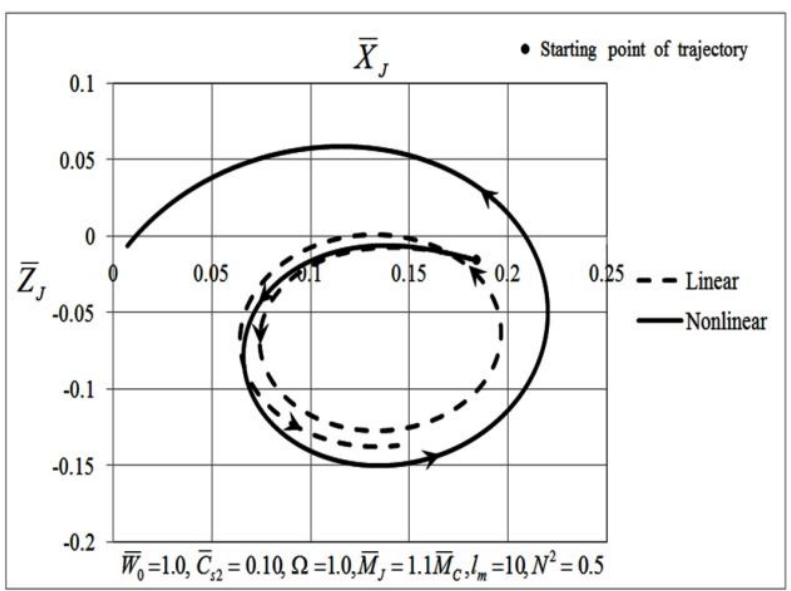

(f)

Fig. 6 Linear and Nonlinear trajectories of journal centre motion for $\bar{W}_{0}=1.0, \quad \bar{C}_{\mathrm{s} 2}=0.10, \quad \Omega=1.0:$ (a) $\bar{M}_{J}=0.80 \bar{M}_{C^{\prime}} \quad$ (b)
$\bar{M}_{I}=\bar{M}_{C^{\prime}}$
(c) $\bar{M}_{I}=1.1 \bar{M}_{C^{\prime}}$
(d) $\bar{M}_{I}=0.80 \bar{M}_{C}, \quad l_{m}=10, \quad N^{2}=0.5$,
(e) $\bar{M}_{I}=\bar{M}_{C}, \quad l_{m}=10$
$N^{2}=0.5$,
(f) $\bar{M}_{I}=1.1 \bar{M}_{C^{\prime}}$
$l_{m}=10, \quad N^{2}=0.5$

for different values of $l_{m}$ and $N^{2}$ while the nonlinear trajectories show stable response in all the cases. The micropolar effect increases as value of $N^{2}$ is increased from 0.5 to 0.9 keeping $l_{m}$ constant at a value of 10 . The influence of micropolar parameters on the journal bearing trajectories is such Japanese Society of Tribologists (http://www.tribology.jp/) that the trajectories start shifting towards origin and a maximum shift is observed at $N^{2}=0.9, l_{m}=10$. This shift signifies that as the micropolar effect increases the journal bearing system stability margin improves with increased load carrying capacity.

Figures 10(a,e) show the influence of $\bar{C}_{S 2}$ (restrictor design 


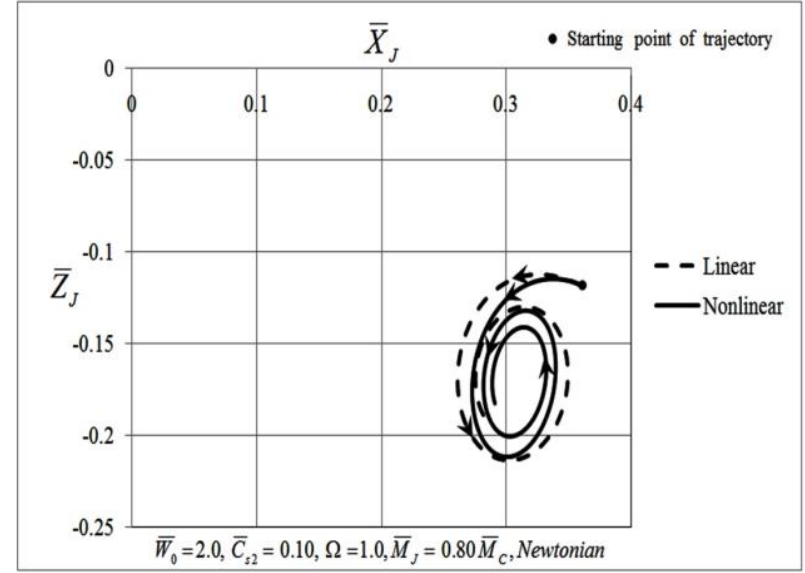

(a)

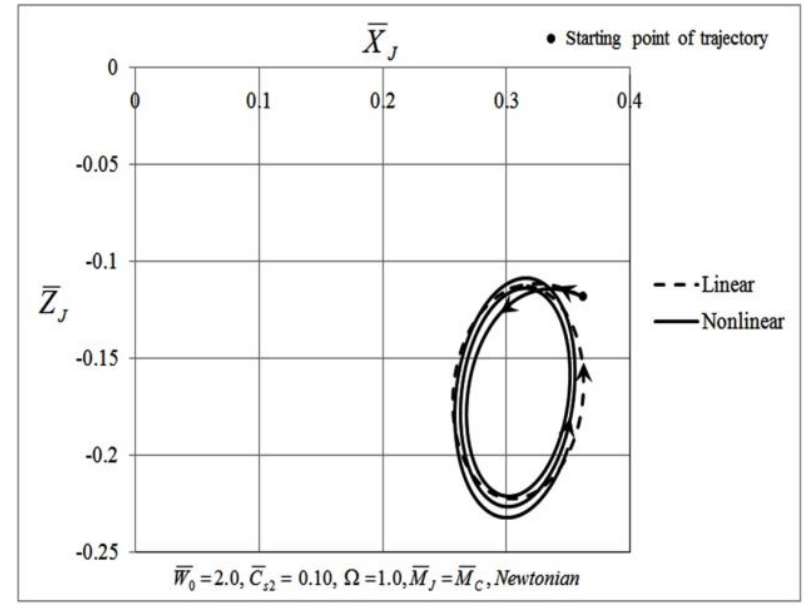

(b)

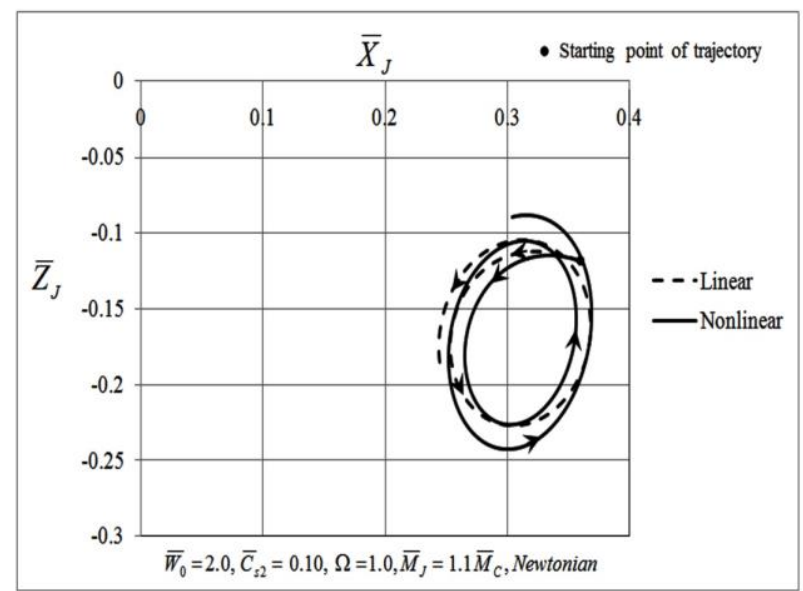

(c)

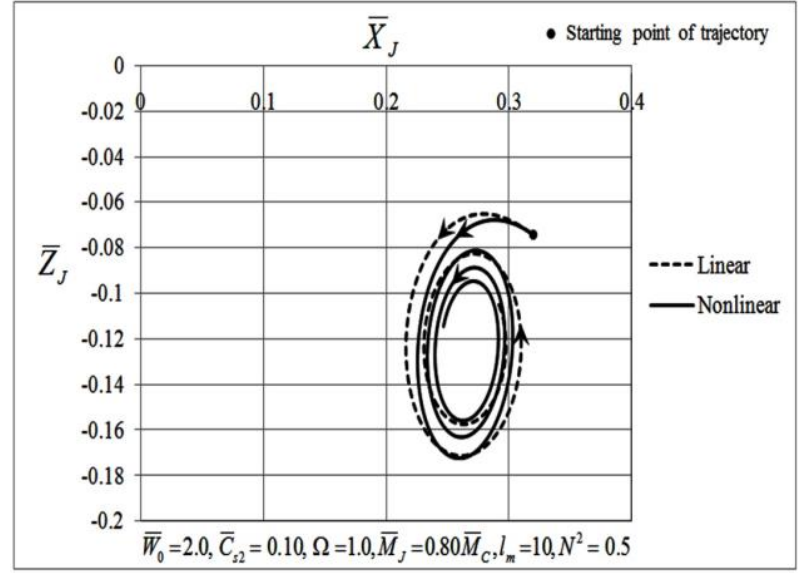

(d)

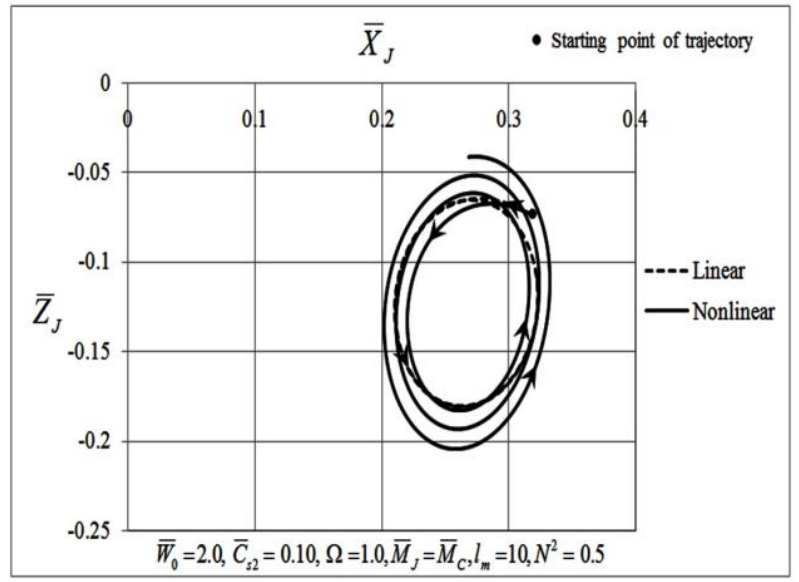

(e)

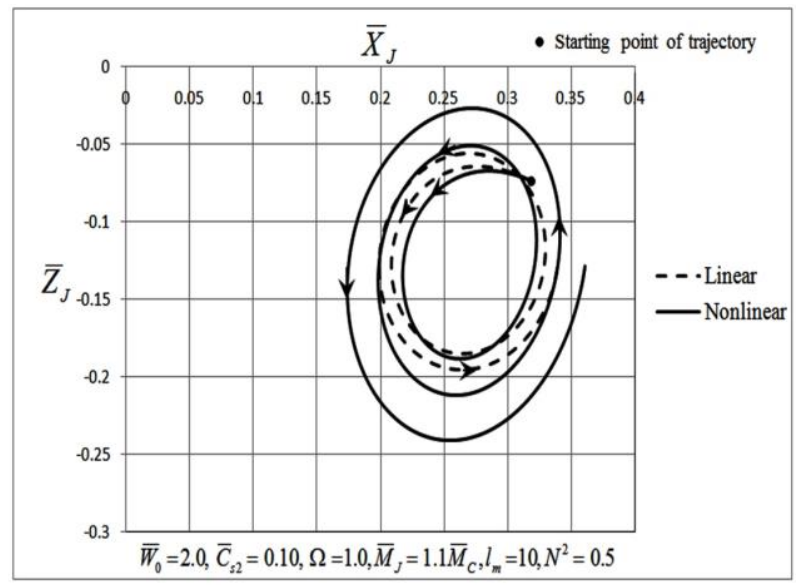

(f)

Fig. 7 Linear and Nonlinear trajectories of journal centre motion for $\bar{W}_{0}=2.0, \quad \bar{C}_{s 2}=0.10, \quad \Omega=1.0:$ (a) $\bar{M}_{J}=0.80 \bar{M}_{C}, \quad(b)$
$\bar{M}_{I}=\bar{M}_{C}$
(c) $\bar{M}_{J}=1.1 \bar{M}_{C^{\prime}}$
(d) $\bar{M}_{I}=0.80 \bar{M}_{C}, \quad l_{m}=10, \quad N^{2}=0.5$,
(e) $\bar{M}_{J}=\bar{M}_{C}, \quad l_{m}=10$
$N^{2}=0.5$
(f) $\bar{M}_{J}=1.1 \bar{M}_{C^{\prime}}$
$l_{m}=10, \quad N^{2}=0.5$

parameter) on the nonlinear journal motion trajectories at $\bar{W}_{0}=2.0, \quad \Omega=1.0, \quad \bar{M}_{I}=\bar{M}_{C^{\prime}} \quad l_{m}=20, \quad N^{2}=0.9 \quad$ for

Newtonian and micropolar lubricants. In these figures, trajectories drawn in red are for Newtonian lubricant and those drawn in blue are for micropolar lubricant. Fig. 10(a) shows that a stable cycle is observed when the lubricant is considered to be Newtonian while the trajectory follows a limit cycle in case of micropolar lubricants for smaller values of $\bar{C}_{S 2}$. Increase in $\bar{C}_{S 2}$ 


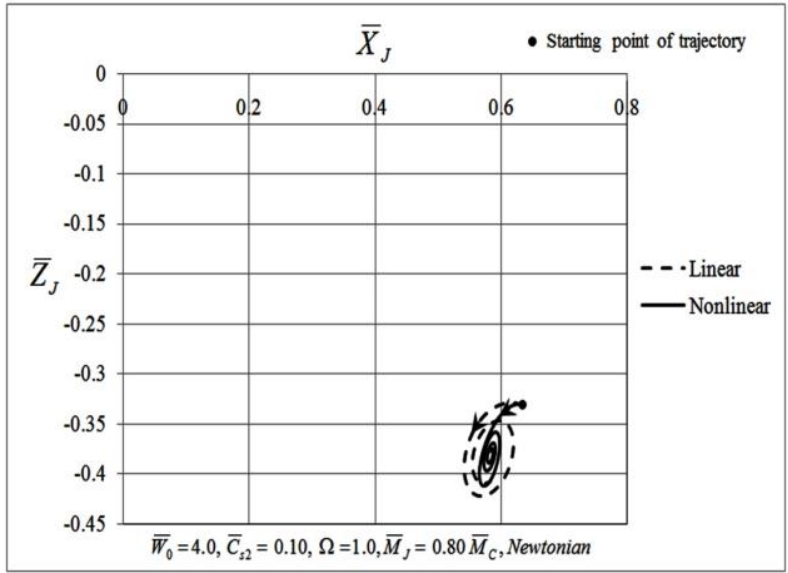

(a)

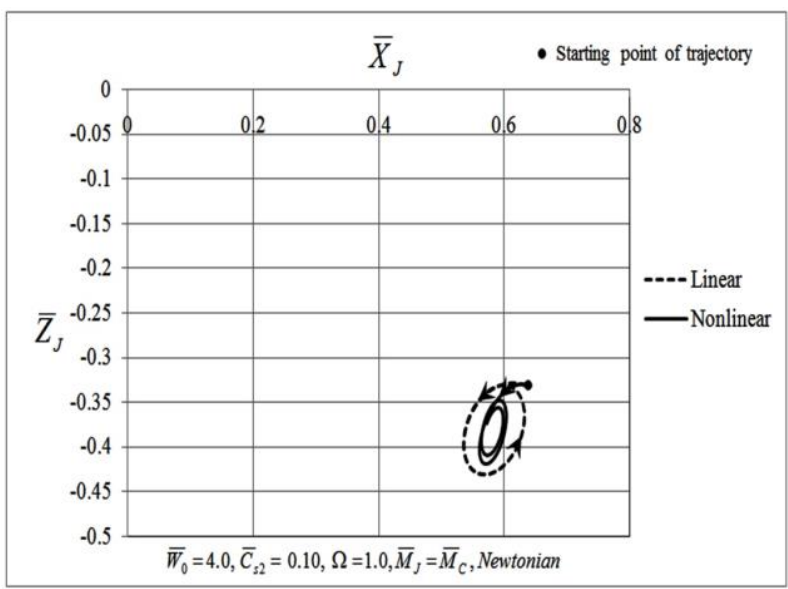

(b)

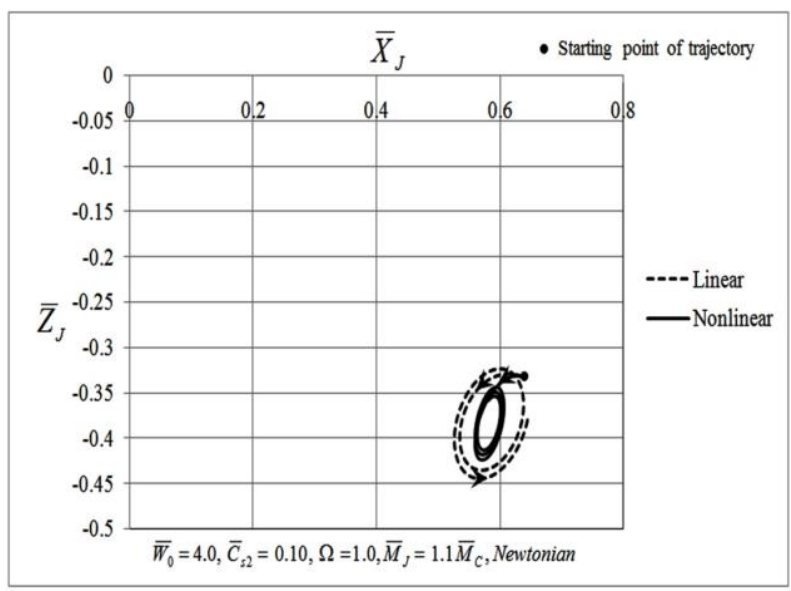

(c)

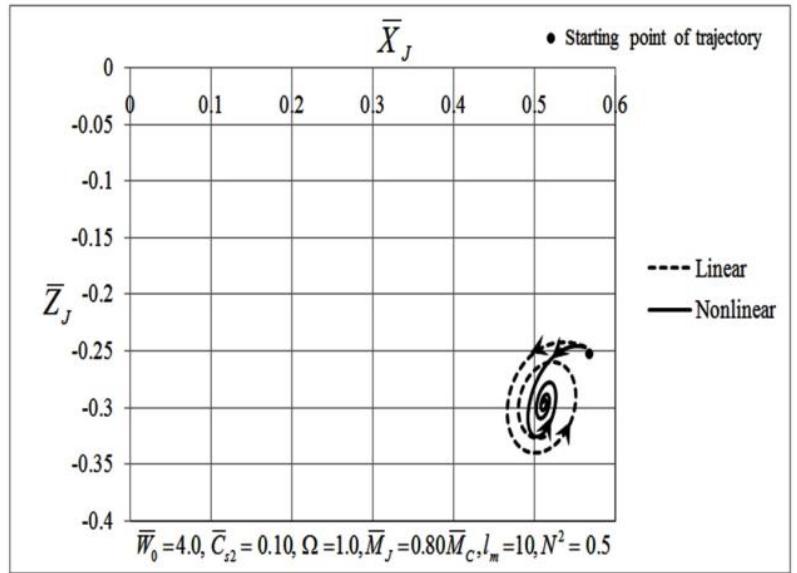

(d)

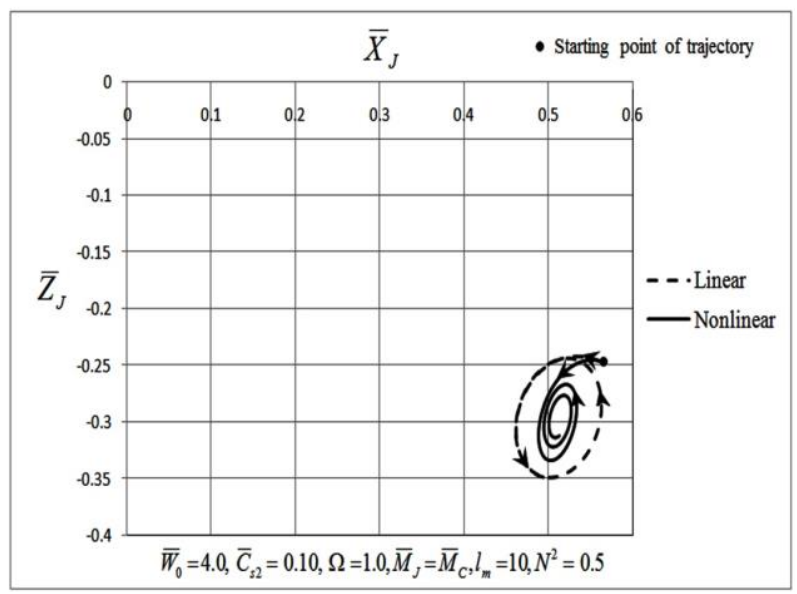

(e)

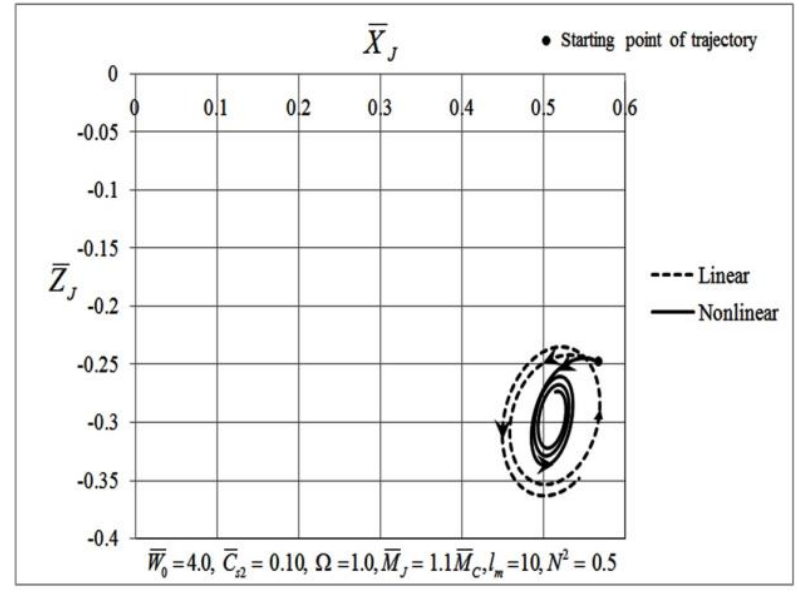

(f)

Fig. 8 Linear and Nonlinear trajectories of journal centre motion for $\bar{W}_{0}=4.0, \quad \bar{C}_{\mathrm{s} 2}=0.10, \quad \Omega=1.0:$ (a) $\bar{M}_{J}=0.80 \bar{M}_{C^{\prime}} \quad$ (b)
$\bar{M}_{J}=\bar{M}_{C^{\prime}}$
(c) $\bar{M}_{J}=1.1 \bar{M}_{C^{\prime}}$
(d) $\quad \bar{M}_{J}=0.80 \bar{M}_{C^{\prime}} \quad l_{m}=10, \quad N^{2}=0.5$,
(e) $\quad \bar{M}_{J}=\bar{M}_{C^{\prime}}, l_{m}=10, \quad N^{2}=0.5$
(f) $\bar{M}_{J}=1.1 \bar{M}_{C^{\prime}}$
$l_{m}=10, \quad N^{2}=0.5$

can occur if the radius of the capillary is increased or length and radial clearance is decreased. Figs. 10(b,c) clearly indicate that as the value of $\bar{C}_{S 2}$ is increased from 0.05 to 0.10 and then from 0.10 to 0.15 , the trajectories show unstable behavior in both the

cases. The increase in the value of $\bar{C}_{S 2}$ causes increase in lubricant flow that leads to the generation of unbalanced forces resulting in instability. When the value of $\bar{C}_{S 2}$ is further increased i. e. at $\bar{C}_{S 2}=0.20$, it is observed that Newtonian orbit 


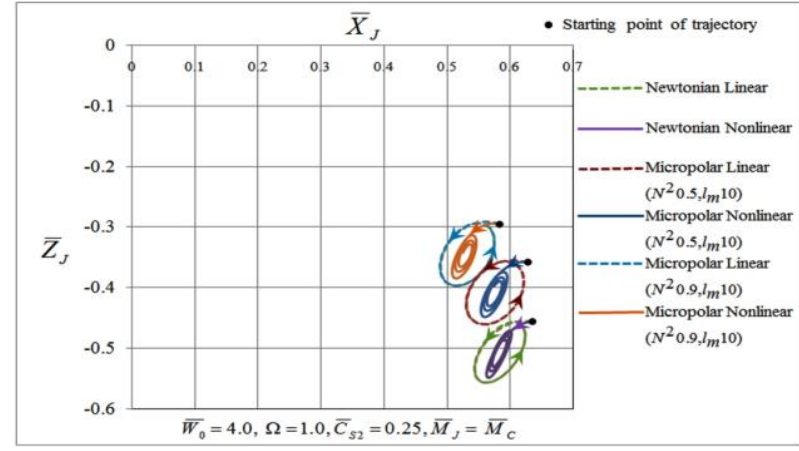

Fig. 9 Linear and Nonlinear Journal Centre Motion Trajectories showing influence of micropolar parameters at $\bar{W}_{0}=4.0, \Omega=1.0, \bar{C}_{52}=0.25$, $\bar{M}_{J}=\bar{M}_{C}$

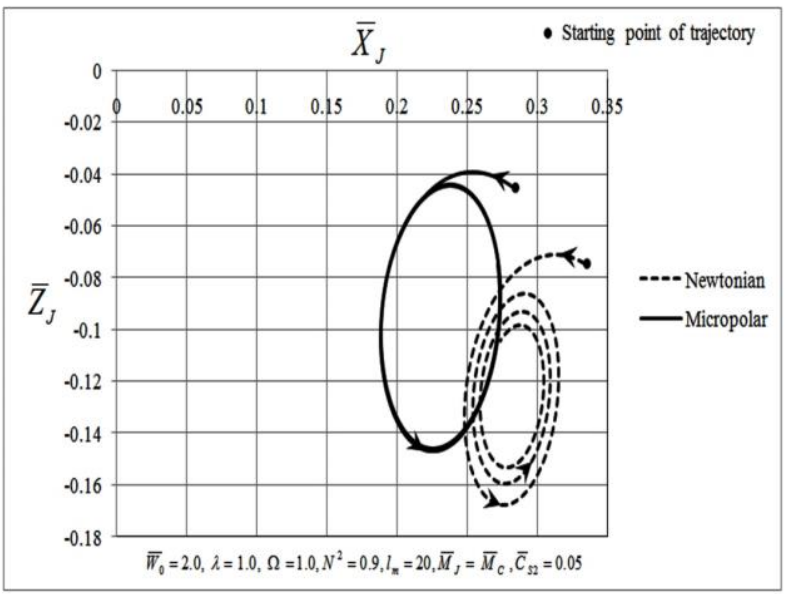

(a)

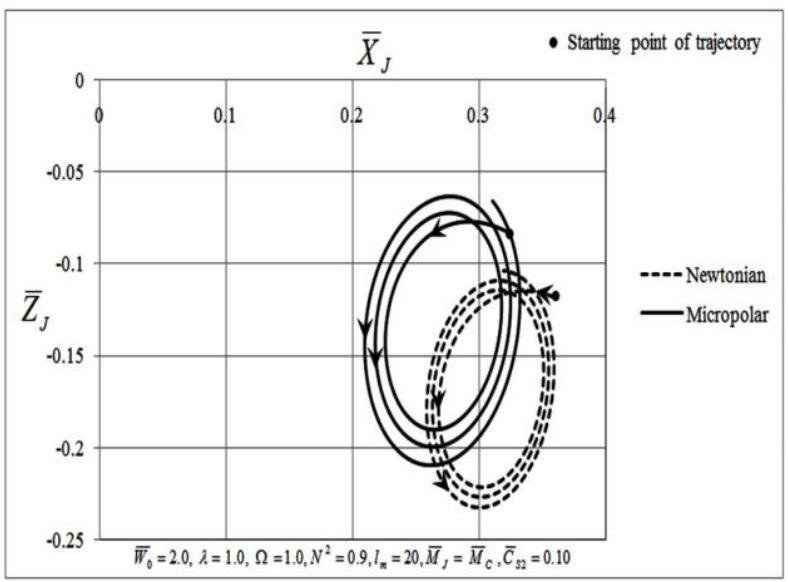

(b)

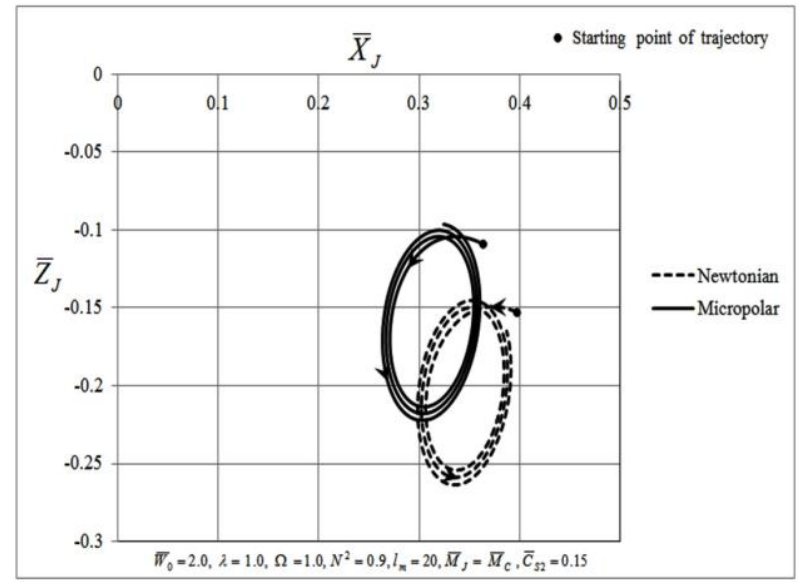

(c)

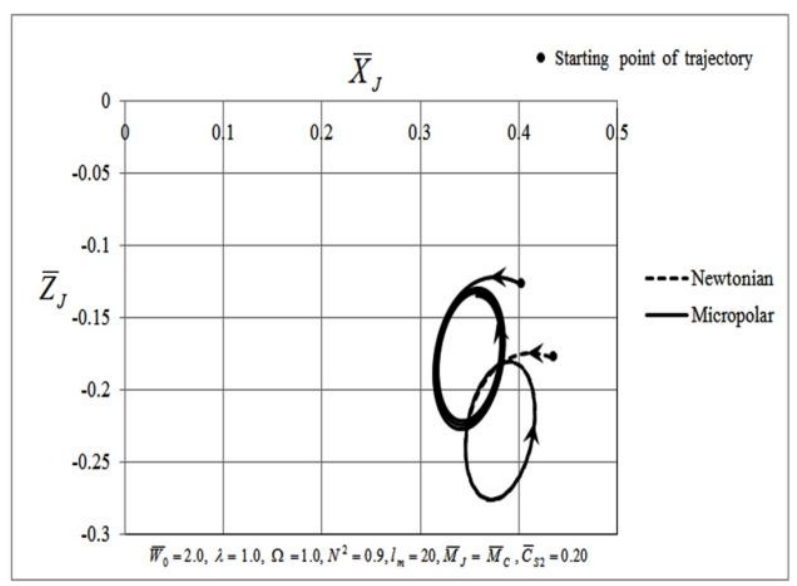

(d)

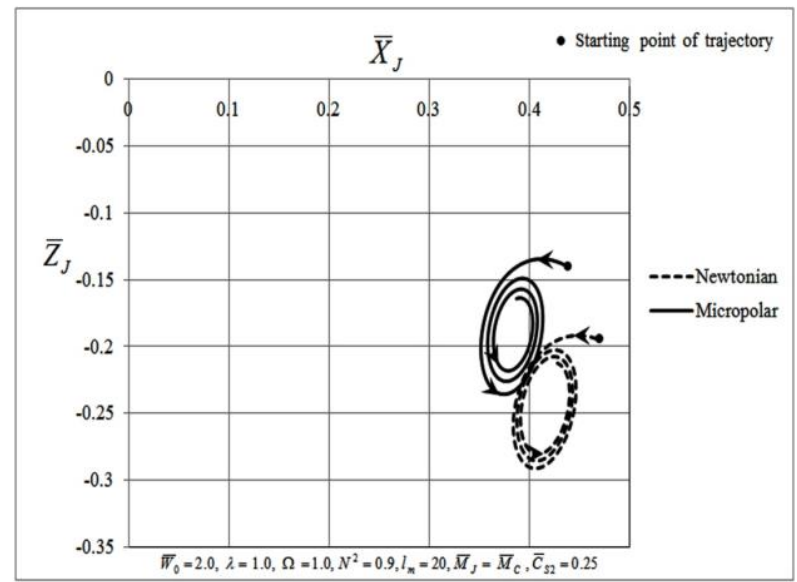

(e)

Fig. 10 Nonlinear trajectories of journal centre motion showing the influence of restrictor design parameter at $\bar{W}_{0}=2.0, \quad \lambda=1.0$,
$\Omega=1.0, \quad N^{2}=0.9 . \quad l_{m}=20, \quad \bar{M}_{I}=\bar{M}_{C}:(\mathrm{a}) \bar{C}_{S 2}=0.05$,
(b) $\bar{C}_{s 2}=0.05$,
(c) $\bar{C}_{S 2}=0.05$,
(d) $\bar{C}_{S 2}=0.05$,
(e) $\bar{C}_{S 2}=0.05$

follows a limit cycle, while the micropolar orbit becomes stable as shown in Fig. 10(d). When the value of $\bar{C}_{S 2}$ is increased till 0.25 , orbits exhibit stable motion for Newtonian as well as micropolar lubricants as shown in Fig. 10(e). Higher values of $\bar{C}_{S 2}$ may be obtained mainly by increasing radius and decreasing the length of the capillary without varying the radial clearance much. As a result, the unbalanced forces are minimum and the journal starts reaching stable state again. It is interesting to note here that journal bearing operating with micropolar lubricant starts reaching stable condition earlier than that operating with Newtonian lubricant. This shows that there is a significant influence of the restrictor design parameter and micropolar parameters on the stability margin of the journal 
motion. Thus, the results dictate that restrictor design parameter must be wisely selected along with the type of lubricant while designing a symmetric hybrid journal bearing system.

The influence of Newtonian and micropolar lubricant on the threshold speed margin $\bar{\omega}_{t h}$ for different values of external loads $\bar{W}_{o}$ at various values of $l_{m}$ and $N^{2}$ has been shown in Fig. 11. It indicates that the value of $\bar{\omega}_{t h}$ decreases at a faster rate as external load is increased from lower to moderate load i. e. $\bar{W}_{o}=1.0$ to $\bar{W}_{o}=2.0$ and then the slope of $\bar{\omega}_{t h}$ almost become constant at heavier loads. This may be due to the fact that between lower and medium loads the increase in critical mass of the journal is less compared to the fluid film reaction produced which causes threshold speed to drop immediately as the load is raised to moderate load from lower load. At lower loads, a maximum of $6.24 \%$ higher threshold stability margin is obtained in case of micropolar lubricant at $N^{2}=0.9$ and $l_{m}=10$ as compared to the journal operating with Newtonian lubricant.

To have a better physical insight on journal motion, the stability threshold speed margin $\overline{\bar{\omega}}_{\text {th }}$ for nonlinear case is presented for symmetrical hole-entry hybrid journal bearing system. It is clearly shown in Figs. 12(a,b) that journal bearing system achieves stability as the load is increased from minimum to maximum. At lower loads, nonlinear trajectory shows instability for both Newtonian as well as micropolar lubricants, but when the load is increased steadily, the journal motion starts exhibiting limit cycle orbits and finally the journal bearing system becomes stable at heavy loads for Newtonian as well as micropolar lubricants. Percentage change in $\overline{\bar{w}}_{t h}$ for symmetric hole-entry hybrid journal bearing system due to the influence of micropolar behavior is shown in Table 4.

Graphical representation of variation of critical mass, $\bar{M}_{C}$ vs load, $\bar{W}_{o}$ for Newtonian and micropolar lubricants indicates physical insight of critical mass change, while the load is being increased. It is observed from Fig. 13, that critical mass decreases initially with increase in load and its value is lowest when load $\bar{W}_{o}$ is 2.0, when the load is further increased from $\bar{W}_{o}=2.0$ to $\bar{W}_{o}=4.0$, a steep increase in critical mass is observed with respect to the load values. So, it may be concluded from here that there exist a certain value of external load, $\bar{W}_{o}$ where the journal critical mass, $\bar{M}_{C}$ is minimum. A maximum of $12 \%$ change in critical mass is observed in case of micropolar lubricant at $\Omega=1.0, \quad \bar{C}_{S 2}=0.15, \lambda=1.0, \bar{a}_{b}=0.25, \quad l_{m}=10$,

$N^{2}=0.9$ compared to Newtonian lubricant under same operating conditions. Percentage change in critical mass due to micropolar behavior is indicated in Table 5 .

To analyze the trend of critical mass in accordance with change in restrictor design parameter values, a graph displaying the variation of critical mass, $\bar{M}_{C}$ vs restrictor design parameter, $\bar{C}_{S 2}$, for Newtonian and micropolar lubricants at $\Omega=1.0, \quad \bar{W}_{o}=4.0, \quad \lambda=1.0, \quad \bar{a}_{b}=0.25$ has been plotted to gain a better understanding of the micropolar behavior of the lubricant on the dynamic characteristics and stability of the journal bearing system. The inference drawn from this graph will be insightful for the bearing designer as he will have the

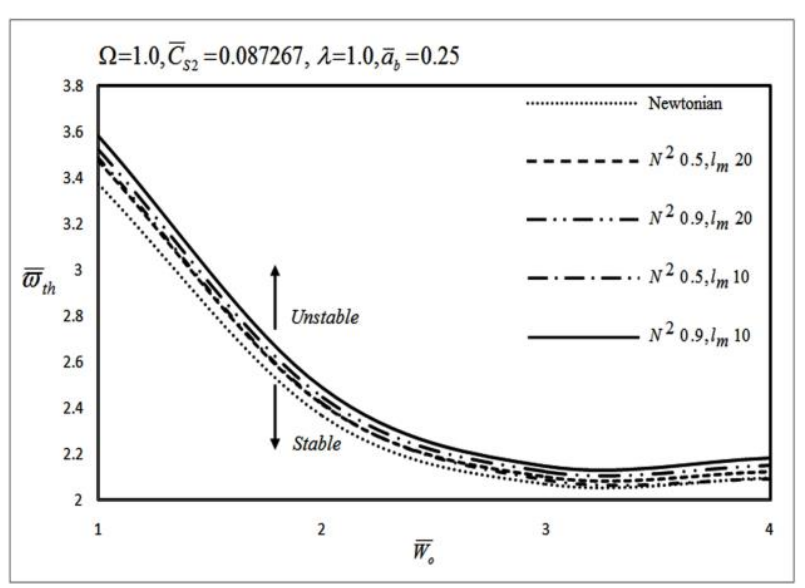

Fig. 11 Variation of $\overline{\bar{w}}_{\text {th }}$ with respect to $\bar{W}_{o}$ for Newtonian and Micropolar Lubricants

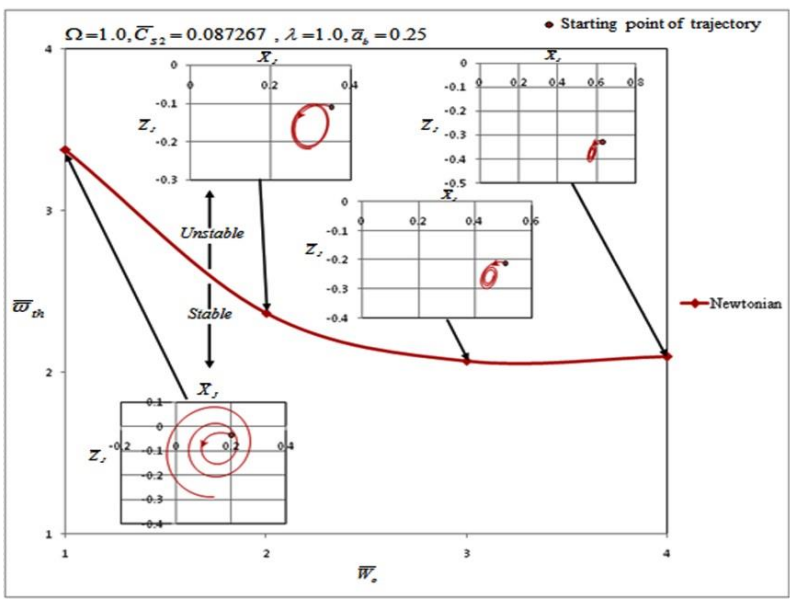

(a)

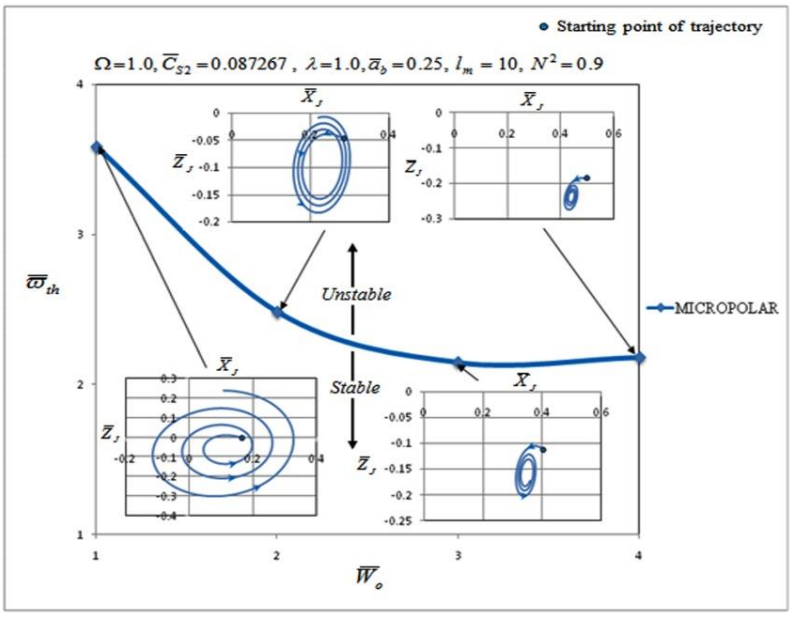

(b)

Fig. 12 (a) Variation of $\overline{\bar{\omega}}_{\text {th }}$ with respect to $\bar{W}_{o}$ for Newtonian Lubricant at $\Omega=1.0, \quad \bar{C}_{S 2}=0.087267$, $\lambda=1.0, \quad \bar{a}_{b}=0.25 \quad$ (b) Variation of $\overline{\bar{\omega}}_{\text {th }}$ with respect to $\bar{W}_{o}$ at $\Omega=1.0, \quad \bar{C}_{S 2}=0.087267$, $\lambda=1.0, \quad \bar{a}_{b}=0.25, \quad l_{m}=10, \quad N^{2}=0.9 \quad$ for Micropolar Lubricant 
information to choose different values of restrictor design parameter for different micropolar lubricants, as critical mass will be known to him at these operating conditions. It is observed from Fig. 14 that critical mass decreases initially with increase in $\bar{C}_{S 2}$ and its value is lowest when $\bar{C}_{S 2}=0.10$; further increase in $\bar{C}_{S 2}$ from $\bar{C}_{S 2}=0.10$ to $\bar{C}_{S 2}=0.25$ causes a steep increase in critical mass with respect to the $\bar{C}_{S 2}$ values. If the radius of the capillary restrictor is increased or length is decreased irrespective of radial clearance, it will result in increased flow which will change the dynamic coefficients i.e. stiffness and damping coefficients leading to increased critical mass of the journal. A maximum of $12 \%$ change in critical mass is observed again in case of bearing system operating with micropolar lubricant at $\Omega=1.0, \bar{W}_{o}=4.0, \lambda=1.0, \quad \bar{a}_{b}=0.25$, $l_{m}=10, \quad N^{2}=0.9$ as compared to Newtonian lubricant under same operating conditions. The percentage change in critical mass due to restrictor design parameter and micropolar parameters is summarized in Table 6 . Comparison of Tables $(5,6)$ yields that the maximum percentage change in critical mass is $12.04 \%$ at $\quad \bar{W}_{o}=4.0, \quad \bar{C}_{S 2}=0.15, \quad N^{2}=0.9, \quad l_{m}=10$.

Figures $15(\mathrm{a}-\mathrm{c})$ shows the variation of eccentricity ratio, $\varepsilon$ with respect to time, $\bar{t}$, for journal bearing system operating with Newtonian and micropolar lubricants at $\Omega=1.0$, $\bar{W}_{o}=4.0, \quad \bar{C}_{s 2}=0.10, \quad \lambda=1.0, \quad \bar{a}_{b}=0.25, \quad N^{2}=0.5, \quad l_{m}=10:$ (a) $\bar{M}_{J}=0.80 \bar{M}_{C^{\prime}} \quad$ (b) $\quad \bar{M}_{J}=\bar{M}_{C^{\prime}}$ (c) $\bar{M}_{J}=1.1 \bar{M}_{C^{\prime}}$. The transient response is quick in nonlinear case as compared to linear case i.e. the system will become stable earlier if nonlinear case is considered at $\bar{M}_{J}=0.80 \bar{M}_{C}$ as shown in Fig. 15(a). It can be seen that with increase in time, as shown in Fig. 15(b), the transient response curves in linear case for both type of

Table 4 Percentage change in $\overline{\bar{\omega}}_{\text {th }}$ for a symmetric hole-entry hybrid journal bearing

\begin{tabular}{|c|c|c|c|c|}
\hline Load $\left(\bar{W}_{o}\right)$ & $N^{2}=0.5 l_{m}=20$ & $N^{2}=0.5 l_{m}=10$ & $N^{2}=0.9 l_{m}=20$ & $N^{2}=0.9 l_{m}=10$ \\
\hline $\mathbf{1}$ & 2.922 & 3.366 & 4.393 & 6.241 \\
\hline $\mathbf{2}$ & 2.090 & 2.313 & 3.416 & 5.090 \\
\hline $\mathbf{3}$ & 1.532 & 0.904 & 2.675 & 3.859 \\
\hline $\mathbf{4}$ & 1.305 & -0.142 & 2.709 & 4.215 \\
\hline
\end{tabular}

$\Omega=1.0, \lambda=1.0, \bar{a}_{b}=0.25, \bar{C}_{s 2}=0.087627$

$\%$ age change $=\frac{\text { Micropolar Values }- \text { Newtonian Values }}{\text { Newtonian Values }} \times 100$

Table 5 Percentage change in $\bar{M}_{C}$ w.r.t. $\bar{W}_{o}$ at $\Omega=1.0, \quad \bar{C}_{S 2}=0.15, \quad \lambda=1.0, \quad \bar{a}_{b}=0.25$

\begin{tabular}{|c|c|c|c|c|}
\hline Load $\left(\bar{W}_{o}\right)$ & $N^{2}=0.5 \quad l_{m}=20$ & $N^{2}=0.5 \quad l_{m}=10$ & $N^{2}=0.9 l_{m}=20$ & $N^{2}=0.9 l_{m}=10$ \\
\hline $\mathbf{1 . 0}$ & 0.8376 & -2.0960 & 1.4240 & -1.9685 \\
\hline $\mathbf{2 . 0}$ & 1.0726 & -1.2930 & 1.8233 & -0.3983 \\
\hline $\mathbf{3 . 0}$ & 3.6121 & 1.9137 & 6.5579 & 10.0887 \\
\hline $\mathbf{4 . 0}$ & 1.3673 & -0.2618 & 5.3652 & 12.0496 \\
\hline
\end{tabular}

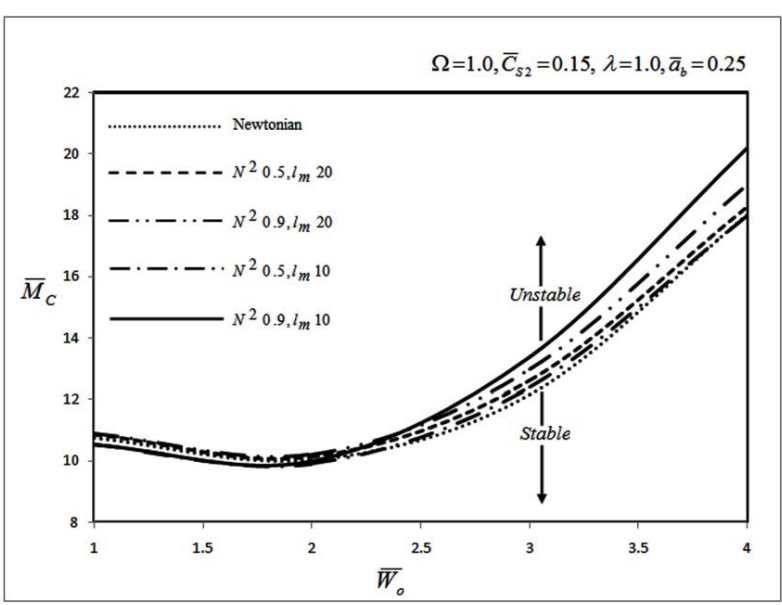

Fig. 13 Variation of $\bar{M}_{C}$ with respect to $\bar{M}_{C}$ for Newtonian and Micropolar Lubricants at $\Omega=1.0$, $\bar{C}_{S 2}=0.15, \lambda=1.0, \quad \bar{a}_{b}=0.25$

Japanese Society of Tribologists (http://www.tribology.jp/)

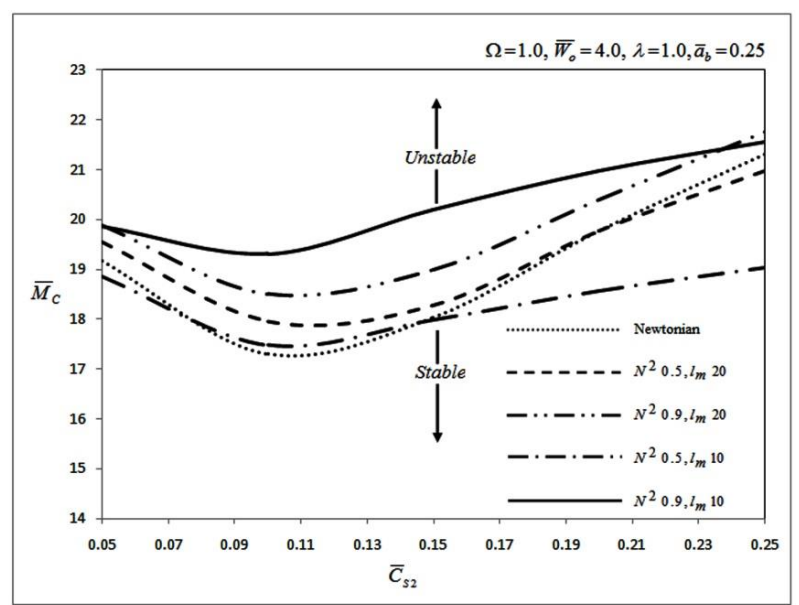

Fig. 14 Variation of $\bar{M}_{C}$ with respect to $\bar{C}_{S 2}$ for Newtonian and Micropolar Lubricants at $\Omega=1.0$, $\bar{W}_{o}=4.0, \lambda=1.0, \quad \bar{a}_{h}=0.25$ 
Table 6 Percentage change in $\bar{M}_{C}$ w.r.t. $\bar{C}_{s 2}$ at $\Omega=1.0, \quad \bar{W}_{o}=4.0, \quad \lambda=1.0, \quad \bar{a}_{b}=0.25$

\begin{tabular}{|c|c|c|c|c|}
\hline $\bar{C}_{S 2}$ & $N^{2}=0.5 \quad l_{m}=20$ & $N^{2}=0.5 \quad l_{m}=10$ & $N^{2}=0.9 \quad l_{m}=20$ & $N^{2}=0.9 \quad l_{m}=10$ \\
\hline $\mathbf{0 . 0 5}$ & 1.9518 & -1.5992 & 3.7003 & 3.5501 \\
\hline $\mathbf{0 . 1}$ & 3.7844 & 1.0503 & 6.9769 & 11.6157 \\
\hline $\mathbf{0 . 1 5}$ & 1.3673 & -0.2618 & 5.3652 & 12.0496 \\
\hline $\mathbf{0 . 2}$ & 0.0323 & -6.0709 & 3.1824 & 6.0846 \\
\hline $\mathbf{0 . 2 5}$ & -1.5147 & -10.6555 & 2.1000 & 1.2293 \\
\hline
\end{tabular}

$\Omega=1.0, \bar{W}_{o}=4.0, \bar{C}_{S 2}=0.10, \lambda=1.0, \bar{a}_{b}=0.25, N^{2}=0.5, l_{m}=10, \bar{M}_{J}=0.80 \bar{M}_{C}$

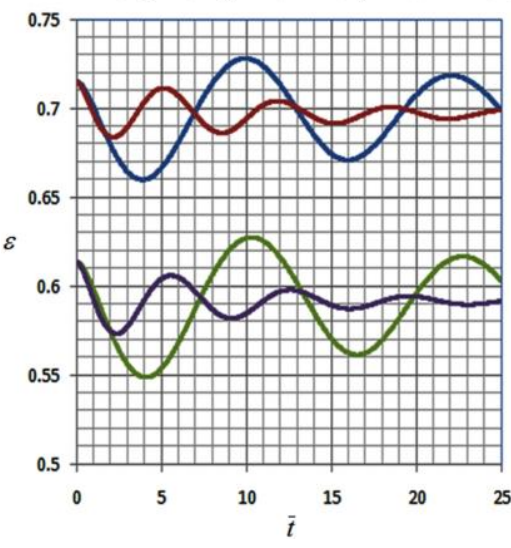

(a)

$\Omega=1.0, \bar{W}_{o}=4.0, \bar{C}_{S 2}=0.10, \lambda=1.0, \bar{a}_{b}=0.25, N^{2}=0.5, l_{m}=10, \bar{M}_{J}=\bar{M}_{C}$

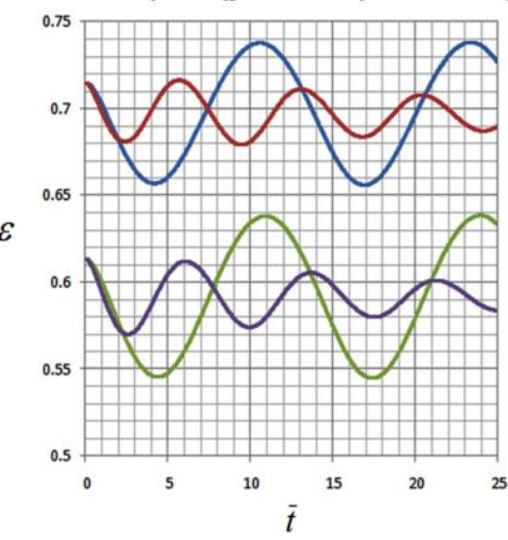

- NEWTONIAN LINEAR

- NEWTONIAN NONLINEAR

- MICROPOLAR LINEAR

- MICROPOLAR NONLINEAR

(b)

lubricants show neither stability nor instability at $\bar{M}_{I}=\bar{M}_{C^{\prime}}$ but for nonlinear case the response curves approach stability with a slow response. Even the transient response predicts instability for linear case at $\bar{M}_{J}=1.1 \bar{M}_{C}$, while a much delayed response towards stability is observed for nonlinear case as shown in Fig. 15(c).

In Fig. 16, the effects of the variation of restrictor design parameter along with micropolar parameters on the frequency of whirling motion of journal center around its steady-state equilibrium point are graphically presented. The results show that there is a gradual decrease in frequency of whirl with an increase in the value of restrictor design parameter for a given load $\bar{W}_{o}$. Also, it is clearly visible from the plot that increase in micropolar effect of the lubricant causes the increase in whirl

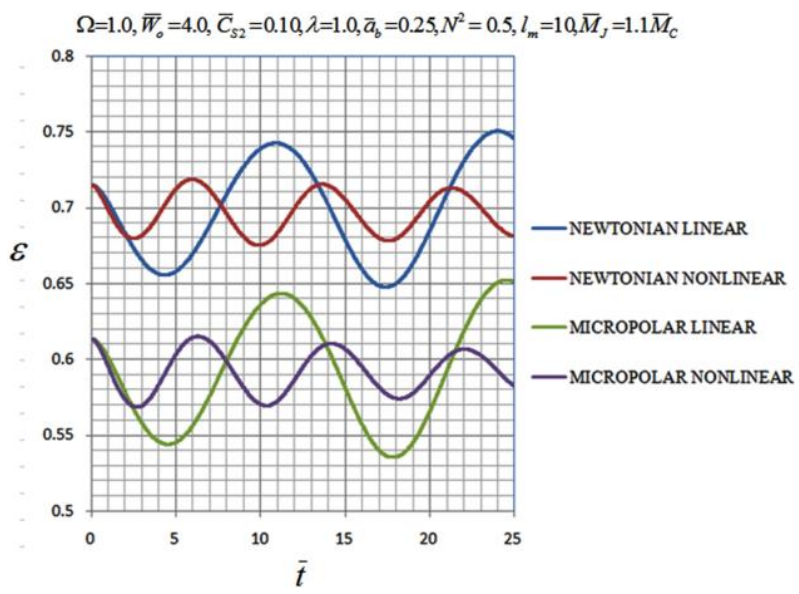

(c)

Fig. 15 Graphs showing the variation of eccentricity ratio, $\varepsilon$ with respect to time, $\bar{t}$ for Newtonian and micropolar lubricants at $\Omega=1.0, \quad \bar{W}_{o}=4.0$, $\bar{C}_{s 2}=0.10, \quad \lambda=1.0, \quad \bar{a}_{b}=0.25, \quad N^{2}=0.5, \quad l_{m}=10$ : (a) $\bar{M}_{J}=0.80 \bar{M}_{C^{\prime}} \quad$ (b) $\bar{M}_{J}=\bar{M}_{C^{\prime}} \quad$ (c) $\bar{M}_{J}=1.1 \bar{M}_{C}$

frequency.

While plotting the frequency of whirl against load, it is observed that frequency of whirl reduces up to moderate loads and then a steep rise in it may be seen at heavy loads for a constant value of restrictor design parameter. The frequency of whirl is found to be minimum between the range of load $\bar{W}_{o}=2.5$ to 3.0 as shown in Fig. 17. Thus, it gives a key idea to the bearing designer to design the bearing for safe loads. In case of micropolar lubricants, it is also noted that for a given coupling number, increase in characteristic length of the lubricant particles causes increase in whirl frequency at higher loads. Also, for a given characteristic length of micropolar lubricant, an increase in coupling number increases the whirl frequency at higher loads above $\bar{W}_{o}=3.0$. 


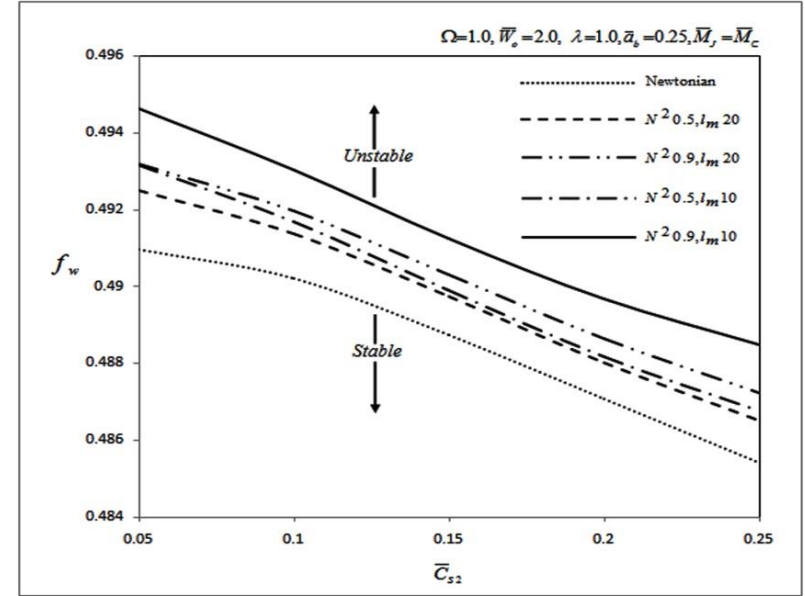

Fig. 16 Variation of frequency of whirl, $f_{w}$ with restrictor

$$
\begin{aligned}
& \text { design parameter } \quad \bar{C}_{S 2} \quad \text { at } \Omega=1.0, \quad \bar{W}_{o}=2.0, \\
& \lambda=1.0, \quad \bar{a}_{b}=0.25, \quad \bar{M}_{J}=\bar{M}_{C}
\end{aligned}
$$

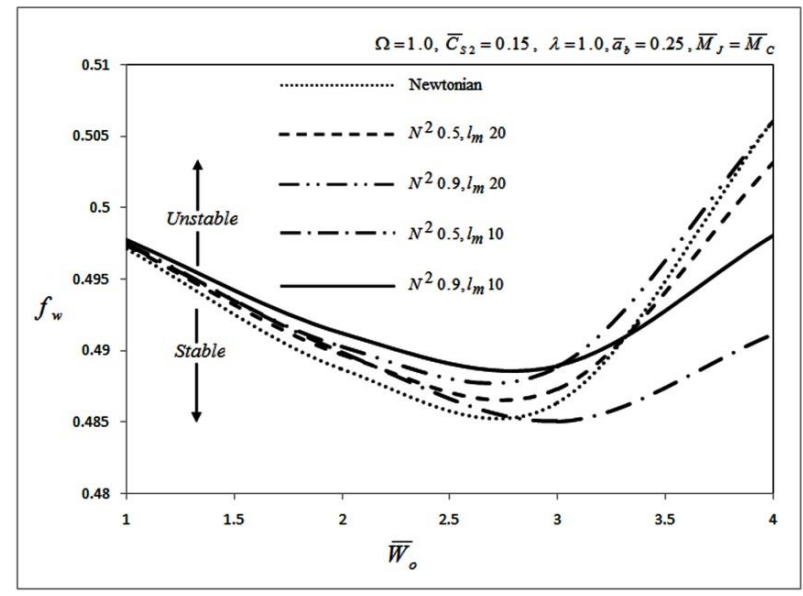

Fig. 17 Variation of frequency of whirl, $f_{w}$ with load $\bar{W}_{o}$

$$
\begin{aligned}
& \text { at: } \quad \Omega=1.0, \quad \bar{C}_{S 2}=0.15, \quad \lambda=1.0, \quad \bar{a}_{b}=0.25, \\
& \bar{M}_{J}=\bar{M}_{C}
\end{aligned}
$$

\section{Conclusions}

The present study deals with influence of micropolar behavior of lubricant on the stability of a rigid and smooth symmetric hole-entry hybrid journal bearing system compensated with capillary restrictor. The linear and nonlinear journal center motion trajectories have been plotted to analyze the stability of the bearing system. A general comparison has been made for both linear and nonlinear trajectories when the journal bearing system is operating with Newtonian and micropolar lubricants. It is observed that the influence of micropolar behavior has a profound effect on linear and nonlinear journal centre motion. As the micropolar effect is increased by increasing $N^{2}$ and decreasing $l_{m}$, the linear and nonlinear journal motion trajectories shifts towards origin as compared to when lubricant is considered as Newtonian. A higher threshold speed margin is obtained for the hybrid journal bearing system operating with micropolar lubricant significantly at lower values of external load. Even increase in micropolar effect of the lubricant causes increase in frequency of whirl at particular value of $\bar{C}_{S 2}$. A decrease in frequency of whirl is observed for increase in $\bar{C}_{S 2}$ values. It is thus inferred that type of lubricant and restrictor design parameter $\bar{C}_{S 2}$ must be chosen wisely as critical mass of the journal, threshold speed margin and frequency of whirl are significantly affected by these.

Comparison of linear and nonlinear models of trajectory motion concludes that nonlinear trajectories give swift and possibly accurate transient response as compared to linear trajectories for Newtonian and micropolar lubricants.

\section{References}

[1] Rowe, W. B., Xu, S. X., Chong, F. S. and Weston, W., "Hybrid Journal Bearing with Particular Reference to Hole-Entry Configurations," Tribology International, 15, 6, 1982, 339-48.

[2] Stout, K. J. and Rowe, W. B., "Externally Pressurized Bearings Design for Manufacture Part 1 Journal Bearing Selection," Tribology International, 7, 3, 1974, 98-106.

[3] Sharma, S. C., Sinhasan, R. and Jain, S. C., “Elastohydrostatic Analysis of Orifice Compensated Multiple Hole-Entry Hybrid Journal Bearings," International Journal of Machine Tools and Manufacture, 30, 1, 1990, 111-129.

[4] Eringen, A. C., "Theory of Micropolar Fluids," Journal of Mathematics and Mechanics, 16, 1, 1966, 1-18.

[5] Das, S., Guha, S. K. and Chattopadhyay, A. K., "On the Steady-State Performance of Misaligned Hydrodynamic Journal Bearings Lubricated with Micropolar Fluids," Tribology International, 35, 4, 2002, 201-210.

[6] Wang, X. L. and Zhu, K. Q., "A Study of the Lubricating Effectiveness of Micropolar Fluids in a Dynamically Loaded Journal Bearing (T1516)," Tribology International, 37, 6, 2004, 481-490.

[7] Wang, X. L. and Zhu, K. Q., "Numerical Analysis of Journal Bearings Lubricated with Micropolar Fluids Including Thermal and Cavitating Effects," Tribology International, 39, 3, 2006, 227-237.

[8] Verma, S., Jadon, V. K. and Gupta, K. D., "Analysis of Capillary Compensated Hydrostatic Journal Bearing Operating with Micropolar Lubricant," Industrial Lubrication and Tribology, 63, 3, 2011, 192-202.

[9] Sharma, S. C. and Ram, N., "Influence of Micropolar Lubricants on the Performance of Slot-Entry Hybrid Journal Bearing," Tribology International, 44, 12, 2011, 1852-1863.

[10] Ram, N. and Sharma, S. C., "Analysis of Orifice Compensated Non-Recessed Hole-Entry Hybrid Journal Bearing Operating with Micropolar Lubricants," Tribology International, 52, 2012, 132-143.

[11] Verma, S., Kumar, V. and Gupta, K. D., "Performance Analysis of Flexible Multirecess Hydrostatic Journal Bearing Operating with Micropolar Lubricant," Lubrication Science, 24, 6, 2012, 273-292.

[12] Verma, S., Kumar, V. and Gupta, K. D., "A Comparative Elastohydrostatic Analysis of CFV and Capillary Compensated Multirecess Hydrostatic/Hybrid Journal Bearing Operating with Micropolar Lubricant," ISRN Tribology, 2013, 924802:17.

[13] Ram, N. and Sharma, S. C., "Influence of Micropolar Lubricants on Asymmetric Slot-Entry Journal Bearings," Tribology Online, 10, 5, 2015, 320-328.

[14] Rana, N. K., Gautam, S. S. and Verma, S., "Comparative Study on the Effect of Recesses on Conical Hybrid Journal Bearing Compensated with CFV under Micropolar Fluid Lubrication," Tribology Online, 11, 3, 2016, 474-486.

[15] Sinhasan. R. and Goyal, K. C., "Transient Response of a Two-Lobe Journal Bearing Lubricated with Non-Newtonian Lubricant," Tribology International, 28, 4, 1995, 233-239.

[16] Andreas, L. S., "Transient Response of Externally Pressurized Fluid Film Bearings," Tribology Transactions, 40, 1, 1997, 147-155.

[17] Turaga, R., Sekhar, A. S. and Majumdar, B. C., “Non-Linear Transient Stability Analysis of a Rigid Rotor Supported on Hydrodynamic 
Journal Bearings with Rough Surfaces," Tribology Transactions, 43, 3, 2000, 447-452.

[18] Das. S., Guha, S. K. and Chattopadhyay, A. K., "Linear Stability Analysis of Hydrodynamic Journal Bearings under Micropolar Lubrication," Tribology International, 38, 5, 2005, 500-507.

[19] Nagaraju, T., Sharma, S. C. and Jain, S. C., "The Stability Margin of a Roughened Hole-Entry Hybrid Journal Bearing System," Tribology Transactions, 48, 1, 2005, 140-146.

[20] Chang-Jian, C. W. and Chen, C. K., "Nonlinear Dynamic Analysis of a Flexible Rotor Supported by Micropolar Fluid Film Journal Bearings," International Journal of Engineering Science, 44, 15-16, 2006, 1050-1070.

[21] Kushare, P. B. and Sharma, S. C., “Nonlinear Transient Stability Study of Two Lobe Symmetric Hole Entry Worn Hybrid Journal Bearing Operating with Non-Newtonian Lubricant," Tribology International, 69, 2014, 84-101.

[22] Sharma, S. C. and Kushare, P. B., "Two Lobe Non-Recessed Roughened Hybrid Journal Bearing- A Comparative Study," Tribology International, 83, 2015, 51-68.

[23] Nagaraju, T., Sharma, S. C. and Jain, S. C., "Study of Orifice Compensated Hole-Entry Hybrid Journal Bearing Considering Surface Roughness and Flexibility Effects," Tribology International, 39, 7, 2006, 715-725.

[24] Mehrjardi, M. Z., Rahmatabadi, A. D. and Meybodi, R. R., "A Comparative Study of the Preload Effects on the Stability Performance of Noncircular Journal Bearings Using Linear and Nonlinear Dynamic Approaches," Journal of Engineering Tribology, $230,7,2016,797-816$.

\section{Nomenclature}

\section{Dimensional Parameters}

\begin{tabular}{|c|c|}
\hline$a$ & Radius of capillary (m) \\
\hline$a_{b}$ & Bearing Land Width in axial direction (m) \\
\hline$c$ & Radial Clearance (m) \\
\hline$C_{i j}$ & Damping Coefficients $(i, j=X, Z) \quad(\mathrm{Ns} / \mathrm{m})$ \\
\hline$D$ & Journal Diameter (m) \\
\hline$e$ & Journal Eccentricity (m) \\
\hline$F$ & Fluid-Film Reaction (N) \\
\hline$F_{o}$ & Resultant Fluid-Film Reaction (N) \\
\hline$F_{o x}, F_{o z}$ & $x$ and $z$ components of Fluid-Film Reaction $(\mathrm{N})$ \\
\hline$g$ & Acceleration due to gravity $\left(\mathrm{m} / \mathrm{s}^{2}\right)$ \\
\hline$h$ & Nominal Fluid-Film Thickness (m) \\
\hline$h_{\min }$ & Minimum Fluid-Film Thickness (m) \\
\hline$L$ & Bearing Length (m) \\
\hline$l=\left(\frac{\gamma}{4 \mu}\right)^{\frac{1}{2}}$ & Characteristic Length (m) \\
\hline$l_{\text {cap }}$ & Capillary Length (m) \\
\hline$M_{J}$ & Journal Mass (kg) \\
\hline$n$ & Number of nodes \\
\hline$N_{o}$ & Journal Speed (RPM) \\
\hline$p$ & Pressure $\left(\mathrm{N} / \mathrm{m}^{2}\right)$ \\
\hline$p_{s}$ & Supply Pressure $\left(\mathrm{N} / \mathrm{m}^{2}\right)$ \\
\hline$p_{\max }$ & Maximum Pressure $\left(\mathrm{N} / \mathrm{m}^{2}\right)$ \\
\hline$Q$ & Bearing Lubricant Flow $\left(\mathrm{m}^{3} / \mathrm{s}\right)$ \\
\hline$R_{J}$ & Journal Radius (m) \\
\hline$S_{i j}$ & Stiffness Coefficients $(i, j=X, Z) \quad(\mathrm{N} / \mathrm{m})$ \\
\hline$t$ & Time (s) \\
\hline$U=\omega_{J} R_{J}$ & Journal Surface Speed (m/s) \\
\hline
\end{tabular}

$\begin{array}{ll}W_{o} & \text { External Load }(\mathrm{N}) \\ X, Y, Z & \text { Cartesian Co-ordinates } \\ X_{J}, Z_{I} & \text { Journal Centre Co-ordinates at steady state in } \\ & \text { equilibrium }(\mathrm{m}) \\ x, y & \text { Circumferential and Axial Coordinates }(\mathrm{m}) \\ z & \text { Coordinate across the Film Thickness } \\ O_{I}, O_{B} & \text { Journal and Bearing Centers } \\ \omega_{I}=\left(\frac{g}{c}\right)^{\frac{1}{2}} & \end{array}$

\section{Greek Symbols}

$\gamma$

$\kappa$

$\lambda$

$\mu \quad$ Dynamic Viscosity of Lubricant $(\mathrm{Pa} \cdot \mathrm{s})$

$\mu_{r} \quad$ Reference Dynamic Viscosity of Lubricant (Pa $\left.\cdot \mathrm{s}\right)$

$\phi \quad$ Attitude Angle

$\omega_{J} \quad$ Journal Rotational Speed ( $\left.\mathrm{rad} / \mathrm{s}\right)$

$\omega_{\text {th }} \quad$ Threshold Speed (rad/s)

$\Gamma, \Omega_{d} \quad$ Solution Boundary/Domain

\section{Non-Dimensional Parameters}

$\bar{a}_{b}=\frac{a_{b}}{L}$

$\bar{c}=\frac{c}{R_{J}}$

$\bar{C}_{i j}=C_{i j}\left(\frac{p_{s} R_{I}}{E_{b} c}\right)$

$\bar{C}_{s 2}=\frac{\pi a^{4}}{8 c^{3} l_{c a p}}=$ Capillary Restrictor Design Parameter

$\bar{F}=F\left(\frac{1}{p_{s} R_{J}^{2}}\right)$

$\bar{h}, \bar{h}_{\min }=\frac{\left(h, h_{\text {min }}\right)}{c}$

$l_{m}=\frac{c}{l}=$ Non-Dimensional Characteristic Length

$\bar{M}_{J}=M_{J}\left(\frac{c g}{p_{s} R_{J}^{3}}\right)$

$N=\left(\frac{\kappa}{2 \mu+\kappa}\right)^{\frac{1}{2}}=$ Coupling Number

$\bar{p}=\frac{p}{p_{s}}$

$\bar{p}^{*}=$ Concentric Pressure Ratio

$\bar{Q}=Q\left[\frac{\mu_{r}}{c^{3} p_{s}}\right]$

$\bar{S}_{i j}=S_{i j}\left[\frac{c}{p_{s} R_{J}^{2}}\right]$ 


$$
\begin{aligned}
& \bar{t}=t\left[\frac{c^{2} p_{s}}{\mu_{r} R_{I}^{2}}\right] \\
& \bar{W}_{o}=\frac{W}{p_{s} R_{J}^{2}} \\
& \alpha, \beta=\frac{(x, y)}{R_{I}} \\
& \bar{z}=\frac{z}{h} \\
& \bar{\mu}=\frac{\mu}{\mu_{r}} \\
& \varepsilon=\frac{e}{c}=\text { Eccentricity Ratio } \\
& \bar{\omega}_{t h}=\left(\frac{\omega_{t h}}{\omega_{I}}\right) \\
& \bar{X}_{I}, \bar{Z}_{J}=\frac{X_{I}, Z_{I}}{c} \\
& f_{w} \text { Frequency of Whirl } \\
& \Omega=\omega_{J}\left(\frac{\mu_{r} R_{I}^{2}}{c^{2} p_{s}}\right)=\text { Speed Parameter }
\end{aligned}
$$

\section{Matrices and Vectors}

$[\bar{C}] \quad$ Fluid-Film Damping Coefficient Matrix

$[\bar{F}] \quad$ Fluidity Matrix

$[\bar{K}] \quad$ System Stiffness Matrix
[M] Journal Mass Matrix
$[N] \quad$ Shape Function Matrix
$\{\bar{p}\} \quad$ Nodal Pressure Vector
$\{\bar{Q}\} \quad$ Nodal Flow Vector
$\left\{\bar{R}_{H}\right\} \quad$ Column Vector due to Hydrodynamic Terms
$\left\{\bar{R}_{x}, \bar{R}_{z}\right\} \quad$ Nodal RHS Vector due to journal center velocity
$[\bar{S}] \quad$ Fluid-Film Stiffness Coefficient Matrix

\section{Subscripts and Superscripts}

$\begin{array}{ll}\text { cap } & \text { Capillary } \\ e & e^{\text {th }} \text { Element } \\ J & \text { Journal } \\ \max & \text { Maximum Value } \\ \min & \text { Minimum Value } \\ o & \text { Steady State Condition } \\ p & \text { Parameter due to pressure } \\ R & \text { Restrictor } \\ r & \text { Reference Value } \\ s & \text { Supply Pressure } \\ x, y, z & \text { Components in } X, Y \text { and } Z \text { directions } \\ * & \text { Concentric Operation } \\ \cdot & \text { First derivative } w . r . t . \text { time } \\ . . & \text { Second derivative } w . r . t . \text { time }\end{array}$

